\title{
DIGITALCOMMONS
}

@WAYNESTATE-

Wayne State University

9-1-2006

\section{Necessary Conditions in Multiobjective Optimization With Equilibrium Constraints}

Truong Q. Bao

Wayne State University

Panjak Gupta

University of Delhi, New Delhi, India

Boris S. Mordukhovich

Wayne State University, boris@math.wayne.edu

\section{Recommended Citation}

Bao, Truong Q.; Gupta, Panjak; and Mordukhovich, Boris S., "Necessary Conditions in Multiobjective Optimization With Equilibrium Constraints" (2006). Mathematics Research Reports. Paper 39.

http://digitalcommons.wayne.edu/math_reports/39 


\section{NECESSARY CONDITIONS IN MULTIOBJECTIVE OPTIMIZATIONWITH EQUILIBRIUM CONSTRAINTS}

T. Q. BAO, P. GUPTA, and B. S. MORDUKHOVICH

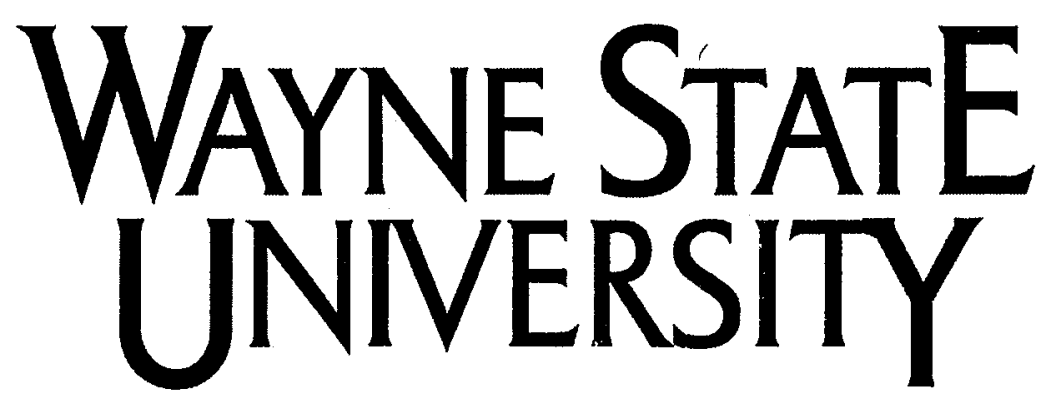

Detroit, MI 48202

Department of Mathematics

Research Report

\section{Series}

$\# 9$

This research was partly supported by the National Science Foundation and the Australian Research Council 


\title{
NECESSARY CONDITIONS IN MULTIOBJECTIVE OPTIMIZATION WITH EQUILIBRIUM CONSTRAINTS ${ }^{1}$
}

\author{
T. Q. BAO 2 , P. GUPTA ${ }^{3}$ AND B. S. MORDUKHOVICH ${ }^{4}$
}

\begin{abstract}
In this paper we study multiobjective optimization problems with equilibrium constraints (MOECs) described by generalized equations in the form

$$
0 \in G(x, y)+Q(x, y)
$$

where both mappings $G$ and $Q$ are set-valued. Such models particularly arise from certain optimization-related problems governed by variational inequalities and first-order optimality conditions in nondifferentiable programming. We establish verifiable necessary conditions for the general problems under consideration and for their important specifications using modern tools of variational analysis and generalized differentiation. The application of the obtained necessary optimality conditions is illustrated by a numerical example from bilevel programming with convex while nondifferentiable data.
\end{abstract}

Key Words: Variational analysis, nonsmooth and multiobjective optimization, variational inequalities, equilibrium constraints, bilevel programming, necessary optimality conditions, generalized differentiation.

\section{Introduction}

This paper is devoted to the study of necessary optimality conditions of an important class of constrained optimization problems called multiobjective optimization with equilibrium constraints (MOECs), which extend various other classes of optimization-related problems well recognized in optimization theory and applications. When there is only one objective, MOECs reduce to the so-called mathematical programs with equilibrium constraints (MPECs), which cover a variety of models particularly arising in applications to problems of operations research, engineering, economics, etc. The reader can find more information in the books from Refs. 1-7 and the bibliographies therein.

A general class of MOECs can be written in the form

$$
\begin{array}{ll}
\operatorname{minimize} & f(x, y) \\
\text { subject to } & \varphi_{i}(x, y) \leq 0, \quad i=1, \ldots, m, \\
& \varphi_{i}(x, y)=0, \quad i=m+1, \ldots, m+r, \\
& (x, y) \in \Omega, \\
& y \in S(x),
\end{array}
$$

\footnotetext{
${ }^{1}$ The main part of this research was conducted during of the visit of the second author at Wayne State University as BOYSCAST Fellow.

${ }^{2}$ Department of Mathematics, Wayne State University, Detroit, Michigan 48202. Research of this author was partially supported by the US National Science Foundation under grant DMS-0304989.

${ }^{3}$ Department of Mathematics, Deen Upadhyaya College, University of Delhi, Shivaji Marg, Karampura, New Delhi-110015, India.

${ }^{4}$ Department of Mathematics, Wayne State University, Detroit, Michigan 48202, USA. Research of this author was partially supported by the US National Science Foundation under grants DMS-0304989 and DMS-0603846 and by the Australian Research Council under grant DP-04511668.
} 
where $f: X \times Y \rightarrow Z$ is a mapping between Banach spaces, $\varphi_{i}: X \times Y \rightarrow \overline{\mathbb{R}}:=(-\infty, \infty]$ are extended-real-valued functions, $\Omega$ is a subset of $X \times Y$, and $S: X \rightrightarrows Y$ is a set-valued mapping describing the so-called equilibrium constraints. The "minimization" in (1) is understood in some multiobjective/vector sense; see below.

The main difference of the MOEC problem (1) from standard mathematical programs and their vector optimization counterparts consists of the presence of the "equilibrium constraint" mapping $S(\cdot)$, which usually describes moving/parameterized sets of optimal and/or equilibrium solutions to parametric problems on the lower hierarchical level. In particular, $S(x)$ in (1) can be sets of optimal solutions and/or KKT (Karush-Kuhn-Tucker) vectors for lower level parametric mathematical programs (which relates to the so-called bilevel programming, or complementarity problems, or variational (hemivariational, quasivariational) inequalities, etc.; see more examples and discussions in the books cited above.

It has been well recognized, starting with the seminal work by Robinson (Ref. 8), that solution sets to the afore-mentioned classes of optimization-related problems, as well as to other models arising in both the theory and applications, can be conveniently described by the so-called parameterized generalized equations in the form

$$
0 \in g(x, y)+Q(y)
$$

with the decision variable $y \in Y$ and the parameter $x \in X$, where $g: X \times Y \rightarrow W$ is a single-valued mapping while $Q: Y \Rightarrow W$ is a set-valued one. In particular, the generalized equation (2) reduces to the parametric variational inequality (VI)

$$
\text { find } y \in \Xi \text { with }\langle g(x, y), u-y\rangle \geq 0 \text { for all } u \in \Xi \subset Y
$$

when $Q(y)=N(y ; \Xi)$ is the normal cone mapping to a convex set. The classical parametric complementarity problem corresponds to (2) when $\Xi$ is the nonnegative orthant in $\mathbb{R}^{n}$. It is well known that the latter model covers sets of optimal solutions with the associated Lagrange multipliers or sets of KKT vectors satisfying first-order necessary optimality conditions in parametric problems of nonlinear programming with smooth data.

A characteristic feature of equilibrium constraints (2) and even simple MPECs associated with them is their intrinsic nonsmoothness, which usually requires the usage of certain tools of generalized differentiation. The reader can find more results and discussions in the above references dealing with various classes of MPECs. We particularly mention the developments in Refs. 9-15 among other publications, where optimality conditions are derived via coderivatives of set-valued mappings introduced by the third author; see his books (Refs. 7,16 ) with more discussions and references therein.

Quite recently, results in this vein have been extended to some problems of multiobjective optimization with several types of equilibrium constraints (2) and preference relations defining vector optimality; see Refs. 7, 17-19. It happens that MOECs are closely connected to a kind of equilibrium problems with equilibrium constraints (EPECs) with Pareto-like preferences on the upper level of hierarchy; see more discussions in Refs. 7, 18-20. Observe also relationships with vector variational inequalities studied, e.g., in Refs. 3,6 .

Note the classical generalized equation model (2), well investigated in optimization theory, does not cover nevertheless some classes of problems important in applications. Let us particularly mention the following two types of equilibrium constraints $S(\cdot)$ in (1):

- $S(x)$ stand for sets of solutions to the so-called set-valued variational inequality (SVI) (called also "generalized variational inequalities"); see, e.g., Ref. 21: given $G: X \times Y \rightrightarrows Y^{*}$ 
and $\Xi \subset Y$, find $y \in \Xi$ such that

$$
\text { there is } y^{*} \in G(x, y) \text { with }\left\langle y^{*}, u-y\right\rangle \geq 0 \text { for all } u \in \Xi \text {. }
$$

Obviously SVI (4) reduces to the standard variational inequality in (3) when the mapping $G=g: X \times Y \rightarrow Y^{*}$ is single-valued.

- Consider the parametric problem of nonsmooth constrained optimization:

$$
\text { minimize } \varphi(x, y) \text { subject to } y \in \Xi \subset Y \text {, }
$$

where $\varphi: X \times Y \rightarrow \bar{R}$ is a lower semicontinuous (l.s.c.) function and where $\Xi$ is a closed set. Then first-order necessary optimality conditions for (5) are written in the form

$$
0 \in \partial_{y} \varphi(x, y)+N(y ; \Xi)
$$

via appropriate subdifferentials of $\varphi$ with respect to $y$ and normal cones to $\Xi$, where $\partial_{y} \varphi$ and $N(\cdot ; \Xi)$ reduce to the corresponding constructions of convex analysis if $\varphi(x, \cdot)$ and $\Xi$ are convex; see, e.g., Ref. 7 for more details. Moreover, the optimality condition (6) in the convex case is known to be not only necessary but also sufficient. In the latter case, the problem of minimizing some function $f(x, y)$ subject to (6) reduces to a nondifferentiable bilevel program; see, e.g., Ref. 4 . When the objective mapping $f: X \times Y \rightarrow Z$ is vector, as in (1), we can call its minimization over (6) a multiobjective bilevel program.

The main difference between (6) and the equilibrium constraints (2) considered before is that the subdifferential mapping $\partial_{y} \varphi(x, y)$ is always set-valued unless $\varphi$ is smooth. It is easy to show the SVI model (4) can also be written in the generalized form (2), where both mappings in the sum are set-valued.

The primary goal of the paper is to derive necessary optimality conditions in constrained problems of multiobjective optimization containing among their constraints equilibrium ones governed by generalized equations in the extended form

$$
0 \in G(x, y)+Q(x, y)
$$

where both $G: X \times Y \Rightarrow W$ and $Q: X \times Y \rightrightarrows W$ are set-valued mappings between Banach spaces. Following the terminology in Ref. 7 (which is used for convenience), we call $G$ the base and $Q$ the field of the generalized equation (7). To our knowledge, equilibrium constraints involving set-valued base mappings $G$ have never been considered explicitly in the literature, even for more special forms in finite-dimensional settings. As seen, they particularly cover models (4) and (6) important for both optimization theory and applications.

Observe one more feature that distinguishes (7) from (2): the field mapping $Q$ in (7) depends not only on the decision variable $y \in Y$ but also on the parameter $x \in X$. The latter model covers, in particular, the class of quasivariational inequalities often arising in applications to mechanics, economics, etc.; see more discussions and examples in Refs. 2, 6. Note that various problems with equilibrium constraints (2) involving $Q=Q(x, y)$ (i.e., those in (7) with single-valued base mappings $G=g: X \times Y \rightarrow W$ ) have been recently studied in Refs. 7, 16, 22; see also the bibliographies therein.

The general class of MOEC problems under consideration in this paper is formulated as 
follows (the "minimization" is understood in the multiobjective sense defined below):

$$
\begin{array}{ll}
\text { minimize } & f(x, y) \\
\text { subject to } & \varphi_{i}(x, y) \leq 0, \quad i=1, \ldots, m, \\
& \varphi_{i}(x, y)=0, \quad i=m+1, \ldots, m+r \\
& (x, y) \in \Omega, \\
& 0 \in G(x, y)+Q(x, y)
\end{array}
$$

where both $G, Q: X \times Y \rightrightarrows W$ are set-valued mappings. We also consider in more detail some important specifications of this general MOEC problem. Most of the results obtained seem to be new not only for (8) and its specifications in the general case of set-valued base mappings $G$ but also when $G=g: X \times Y \rightarrow W$ is single-valued.

The rest of this paper is organized as follows. Section 2 contains basic definitions and preliminaries from variational analysis and generalized differentiation broadly used in the paper. In Section 3 we establish necessary optimality conditions for general MOECs, while Sections 4-6 contain necessary conditions for their various specification including problems of multiobjective fractional programming, multiobjective optimization of set-valued variational inequalities, and certain problems related to bilevel programming. Finally, in Section 7 we illustrate the use of the obtained necessary optimality conditions by considering a numerical example from nondifferentiable bilevel programming.

Thoroughout the paper we employ the standard notation of variational analysis; $c$. the books in Refs. 7 and 23. For a Banach space $X$, we denote its norm by $\|\cdot\|$ and consider the dual space $X^{*}$ equipped with the weak* topology $w^{*}$, where $\langle\cdot, \cdot\rangle$ stands for the canonical pairing between $X$ and $X^{*}$. Given a set-valued mapping $F: X \rightrightarrows X^{*}$, recall that

$$
\begin{array}{r}
\operatorname{Limsup}_{x \rightarrow \bar{x}} F(x):=\left\{x^{*} \in X^{*} \mid \exists \text { sequences } x_{k} \rightarrow \bar{x} \text { and } x_{k}^{*} \stackrel{w^{*}}{\longrightarrow} x^{*}\right. \\
\text { with } \left.x_{k}^{*} \in F\left(x_{k}\right) \text { for all } k \in \mathbb{N}\right\}
\end{array}
$$

signifies the sequential Painlevé-Kuratowski upper/outer limit with respect to the norm topology of $X$ and the weak* topology of $X^{*}$, where $\mathbb{N}:=\{1,2, \ldots\}$. Depending on the context, the symbols $x \stackrel{\Omega}{\rightarrow} \bar{x}$ and $x \stackrel{\varphi}{\rightarrow} \bar{x}$ mean that $x \rightarrow \bar{x}$ with $x \in \Omega$ and $x \rightarrow \bar{x}$ with $\varphi(x) \rightarrow \varphi(\vec{x})$ for a set $\Omega \subset X$ and an extended-real-valued function $\varphi: X \rightarrow \overline{\mathbb{R}}$, respectively.

\section{Basic Tools of Variational Analysis}

In this section we overview some basic tools of variational analysis widely used in formulations and proofs of the main result of the paper. We follow the recent books by Mordukhovich (Refs. 7, 16), where the reader can find more details, discussions, and references. We also refer the reader to the now classical (finite-dimensional) book by Rockafellar and Wets (Ref. 23) and to the recent one by Borwein and Zhu (Ref. 24) for related and additional material on variational analysis, generalized differentiation, and their applications.

Let us begin with reviewing appropriate constructions of generalized differentiation used in what follows. Our main attention in this paper is paid to sets, set/single-valued mappings, and extended-real-valued functions defined in Asplund spaces. Recall that a Banach space $X$ is Asplund if each of its separable subspaces has a separable dual. There are many other equivalent descriptions of the original Asplund property, which can be found, e.g., in Ref. 7 (Chapter 2) and Ref. 25. Observe that every reflexive Banach space is Asplund. 
The following constructions of generalized differentiation enjoy comprehensive calculus properties ("full calculus") in the Asplund space framework. For their useful modifications in general Banach spaces see Refs. 16, 26 and the bibliographies therein.

Let $\Omega \subset X$ be a subset of an Asplund space that is locally closed around $\bar{x} \in \Omega$. Then the (basic, limiting) normal cone to $\Omega$ at $\vec{x}$ is defined by

$$
N(\bar{x} ; \Omega):=\operatorname{Limsup}_{x \rightarrow \bar{x}} \widehat{N}(x ; \Omega)
$$

via the sequential Painlevé-Kuratowski outer limit of the so-called Fréchet normal cone



with $\widehat{N}(x ; \Omega):=\emptyset$ if $x \notin \Omega$. Note that, in contrast to (10), our basic normal cone (9) is often nonconvex enjoying nevertheless full calculus, which is mainly based on the extremal principle; see Ref. 16 for the detailed study and more discussions.

Dealing primarily in this paper with set-valued mappings $F: X \rightrightarrows Y$ of closed graphs

$$
\operatorname{gph} F:=\{(x, y) \in X \times Y \mid y \in F(x)\}
$$

between Asplund spaces, we use the following construction of the (basic, normal) coderivative $D^{*} F(\bar{x}, \bar{y}): Y^{*} \rightrightarrows X^{*}$ of $F$ at $(\bar{x}, \bar{y}) \in \operatorname{gph} F$ defined by

$$
D^{*} F(\bar{x}, \bar{y})\left(y^{*}\right):=\left\{x^{*} \in X^{*} \mid\left(x^{*},-y^{*}\right) \in N((\bar{x}, \bar{y}) ; \operatorname{gph} F)\right\},
$$

which is a positively homogeneous mapping of $y^{*}$; we omit $\bar{y}=f(\bar{x})$ in (11) if $F=f: X \rightarrow Y$ is single-valued. If $f: X \rightarrow Y$ happens to be strictly differentiable at $\bar{x}$ (which is automatic when it is $C^{1}$ around this point), then

$$
D^{*} f(\bar{x})\left(y^{*}\right)=\left\{\nabla f(\bar{x})^{*} y^{*}\right\} \text { for all } y^{*} \in Y^{*} .
$$

In Ref. 16, the reader can find equivalent descriptions of the coderivative (11) and its numerous properties including comprehensive calculus rules and computations for various classes of mappings in finite and infinite dimensions.

Consider further an extended-real-valued function $\varphi: X \rightarrow \overline{\mathbb{R}}$ finite at $\bar{x}$ and lower semicontinuous (l.s.c.) around this point, and let $E_{\varphi}: X \rightrightarrows \mathbb{R}$ be the epigraphical multifunction

$$
E_{\varphi}(x):=\{\mu \in \mathbb{R} \mid \mu \geq \varphi(x)\}
$$

associated with $\varphi$. Then we define the basic subdifferential $\partial \varphi(\bar{x})$ and the singular subdifferential $\partial^{\infty} \varphi(\bar{x})$ of $\varphi$ at $\bar{x}$ via the coderivative (11) of its epigraphical multifunction $E_{\varphi}$ at $(\bar{x}, \varphi(\bar{x})) \in \operatorname{gph} E_{\varphi}$ by, respectively,

$$
\partial \varphi(\bar{x}):=D^{*} E_{\varphi}(\bar{x}, \varphi(\bar{x}))(1) \text { and } \partial^{\infty} \varphi(\bar{x}):=D^{*} E_{\varphi}(\bar{x}, \varphi(\bar{x}))(0) .
$$

Again, we refer the reader to the third author's book in Ref. 16 for various analytic representations of both subdifferentials in (12) and extended calculus rules for them. Note that the singular subdifferential in (12) carries some information only for non-Lipschitzian functions, since we always have

$$
\partial^{\infty} \varphi(\bar{x})=\{0\} \text { when } \oint_{\text {is locally Lipschitzian around } \vec{x}}
$$


Observe also the useful representation of the coderivative

$$
D^{*} \varphi(\bar{x})(0)=\partial^{\infty} \varphi(\bar{x}) \cup \partial^{\infty}(-\varphi)(\bar{x}) .
$$

provided that $\varphi$ is continuous around $\bar{x}$.

One of the most important ingredients of variational analysis in infinite-dimensional spaces, in contrast to its finite-dimensional counterpart, is the necessity to impose some "normal compactness" properties, which particularly allow us to perform limiting procedures of deriving nontrivial calculus rules and optimality conditions and which are automatic in finite dimensions. In this paper we employ the following property for locally closed sets comprehensively studied in Ref. 16 . We say that $\Omega \subset X$ is sequentially normally compact (SNC) at $\bar{x} \in \Omega$ if for any sequence $x_{k} \in \Omega$ and $x_{k}^{*} \in \widehat{N}\left(x_{k} ; \Omega\right), k \in \mathbb{N}$, one has

$$
x_{k}^{*} \stackrel{w^{*}}{\rightarrow} 0 \Longrightarrow x_{k}^{*} \rightarrow 0 \text { as } k \rightarrow \infty .
$$

Besides finite dimensions, this property automatically holds for sets that are compactly epiLipschitzian (CEL) around $\bar{x}$ in the sense of Borwein and Strójwas (Ref. 27). In general, the implication (CEL) $\Longrightarrow$ (SNC) may be strict even for convex subcones of Asplund spaces; see Ref. 28 for a detailed study of relationships between SNC and CEL properties. It is important to emphasize that the SNC and related properties of sets and its modifications for mappings enjoy full calculus (in the sense of comprehensive rules for their preservation under various operations performed with sets and mappings), which is mainly based on the extremal principle in variational analysis; see Ref. 16.

We now present the basic version of the (exact) extremal principle for finitely many sets used in this paper; see Chapter 2 of Ref. 16. Given closed subsets $\Omega_{1}, \ldots, \Omega_{n}(n \geq 2)$ of the space $X$, we say that a point $\bar{x} \in \cap_{i=1}^{n} \Omega_{i}$ is locally extremal for the set system $\left\{\Omega_{1}, \ldots, \Omega_{n}\right\}$ if there exist a neighborhood $U$ of $\vec{x}$ and sequences $\left\{a_{i k}\right\} \subset X, i=1, \ldots, n$, such that $a_{i k} \rightarrow 0$ as $k \rightarrow \infty$ and

$$
\bigcap_{i=1}^{n}\left(\Omega_{i}-a_{i k}\right) \cap U=\emptyset \text { for all } k \in \mathbb{N} .
$$

The Extremal Principle. Let $\bar{x}$ be a local extremal point of the set system $\left\{\Omega_{1}, \ldots, \Omega_{n}\right\}$ in the Asplund space X. Assume that all but one of these sets are SNC at $\bar{x}$. Then there are $\left(x_{1}^{*}, \ldots, x_{n}^{*}\right)$ satisfying the relationships

$$
\begin{gathered}
x_{i}^{*} \in N\left(\bar{x} ; \Omega_{i}\right), \quad i=1, \ldots, n, \quad \text { and } \\
x_{1}^{*}+\ldots+x_{n}^{*}=0, \quad\left\|x_{1}^{*}\right\|+\ldots+\left\|x_{n}^{*}\right\|=1 .
\end{gathered}
$$

The extremal principle can be viewed as a variational counterpart of the convex separation theorem for finitely many sets in nonconvex settings. In fact it plays a fundamental role in variational analysis similar to that played by convex separation and equivalent results in convex analysis as well as in its outgrowths and applications; see the books in Refs. 7 and 16 , which fully revolve around the extremal principle and its modifications. In this paper we develop new applications of the extremal principle to the general MOECs formulated above and their important specifications. 


\section{Necessary Optimality Conditions for General MOECs}

In this section we study the general class of multiobjective optimization problems (8) with equilibrium constraints governed by generalized equations in the extended form (7), where the multiobjective/vector optimization is understood in the following sense.

Definition 3.1 (generalized order optimality). Given a cost mapping $f: T \rightarrow Z$ between Banach spaces, an ordering set $\Theta \subset Z$ containing the origin, and a constraint set $\Xi \subset T$, we say that a point $\bar{t} \in T$ is a locally $(f, \Theta)$-OPTIMAL with respect to $\Xi$ if there are a neighborhood $U$ of $\bar{t}$ and a sequence $\left\{z_{k}\right\} \subset Z$ with $\left\|z_{k}\right\| \rightarrow 0$ as $k \rightarrow \infty$ such that

$$
f(t)-f(\bar{t}) \notin \Theta-z_{k} \text { for all } t \in \Xi \cap U \text { and } k \in \mathbb{N} \text {. }
$$

The notion of generalized order optimality from Definition 3.1 is induced by the concept of set extremality presented in Section 2; see Ref. 7 with the comments and bibliography therein. Note that the ordering set $\Theta$ in Definition 3.1 imposes an order/preference relation between $z_{1}, z_{2} \in Z$ defined via $z_{1}-z_{2} \in \Theta$. When $Z=\mathbb{R}^{n}$ and

$$
\Theta=\mathbb{R}_{\leq}^{n}:=\left\{z \in \mathbb{R}^{n} \mid z_{i} \leq 0, i=1, \ldots, n\right\},
$$

the above optimality notion clearly reduces to the standard local Pareto optimality; for $Z=\mathbb{R}$ and $\Theta=(-\infty, 0]$, it is the classical order on real numbers. Note that we do not assume that $\Theta$ is either convex or its interior is nonempty. When $\Theta$ is a convex subcone of $Z$, Definition 3.1 covers the classical notions of Pareto-type optimality/efficiency requiring that there is no $t \in \Xi \cap U$ with $f(t)-f(\bar{t}) \in \mathrm{ri} \Theta$. To see this it suffices to take $z_{k}:=-z_{0} / k$ for $k \in \mathbb{N}$ with some $z_{0} \in$ ri $\Theta$; see Chapter 5 of Ref. 7 for more discussions.

In what follows we study local $(f, \Theta)$-optimal solutions, in the sense of Definition 3.1, to various constrained multiobjective optimization problems containing particularly the equilibrium constraints described above and their remarkable specifications. To derive necessary optimality conditions for the general MOEC problem (8) in the next theorem, we develop the approach from Ref. 7 reducing the reference local optimal solution for the MOEC problem under consideration to a local extremal point of a set system constructed upon the initial data of problem (8) and then employing the extremal principle for finitely many sets formulated in Section 2.

This approach was realized in Ref. 7 for single-valued base mappings $G=g: X \times Y \rightarrow W$ in the equilibrium constraint of (8) by reducing it to the geometric constraint defined by the closed set

$$
\Xi:=\{(x, y) \in X \times Y \mid 0 \in g(x, y)+Q(x, y)\}
$$

and by applying then calculus rules to present the obtained results via the initial data $g$ and $Q$ of the corresponding MOEC. However, the implementation of this approach in the case of MOEC (8) with set-valued base mappings $G$ requires imposing the inner semicontinuity assumption on the solution map

$$
S(x):=\{y \in Y \mid 0 \in G(x, y)+Q(x, y)\}
$$

to the extended generalized equation (7), which is automatic for single-valued bases while seems to be significantly restrictive in the set-valued case of our main interest in this paper, 
even in finite-dimensional settings. To avoid this limitation, we modify the approach from Ref. 7 and deal now with the graphical sets $\operatorname{gph} G$ and $\operatorname{gph} Q$ as with two separate members of the new extremal set system.

Our first result establishes in this way necessary optimality conditions for the general MOEC problem (8) without imposing any constraint qualification.

Theorem 3.2 (necessary optimality conditions for general MOECs). Let $(\bar{x}, \bar{y})$ be a local $(f, \Theta)$-optimal solution to $M O E C(8)$, where all the spaces under consideration are Asplund. Given $\bar{z}:=f(\bar{x}, \bar{y})$ and $\bar{w} \in G(\bar{x}, \bar{y}) \cap(-Q(\bar{x}, \bar{y}))$, suppose that $f$ is continuous around $(\bar{x}, \bar{y})$, that $\varphi_{i}$ are l.s.c. around $(\bar{x}, \bar{y})$ for $i=1, \ldots, m$ and continuous around this point for $i=m+1, \ldots, m+r$, and that $\Omega$ and $\Theta$ are locally closed around $(\bar{x}, \bar{y})$ and 0 , respectively. Assume further that all but one of the sets $\operatorname{gph} f, \Omega \times(\bar{z}+\Theta)$, epi $\varphi_{i}$ for $i=1, \ldots, m, \operatorname{gph} \varphi_{i}$ for $i=m+1, \ldots, m+r, \operatorname{gph} G$, and $\operatorname{gph} Q$ are $S N C$ at $(\bar{x}, \bar{y}, \bar{z})$, $(\bar{x}, \bar{y}, 0),(\bar{x}, \bar{y}, \bar{w})$, and $(\bar{x}, \bar{y},-\bar{w})$, respectively. Then there are nontrivial dual elements

$$
\left(\lambda_{1}, \ldots, \lambda_{m+r}, z^{*}, w^{*}, x^{*}, y^{*}, x_{1}^{*}, y_{1}^{*}, \ldots, x_{m+r}^{*}, y_{m+r}^{*}, x_{G}^{*}, y_{G}^{*}, x_{Q}^{*}, y_{Q}^{*}, x_{\Omega}^{*}, y_{\Omega}^{*}\right) \neq 0
$$

such that the following necessary optimality conditions are satisfied:

$$
\begin{aligned}
& \lambda_{i} \geq 0 \text { for } i=1, \ldots, m ; \quad \lambda_{i} \in \mathbb{R} \text { for } i=m+1, \ldots, m+r \\
& z^{*} \in N(0 ; \Theta), \quad w^{*} \in W^{*} ; \\
& \left(x^{*}, y^{*}\right) \in D^{*} f(\bar{x}, \bar{y})\left(z^{*}\right) ; \\
& \left(x_{i}^{*}, y_{i}^{*}\right) \in D^{*} E_{\varphi_{i}}(\bar{x}, \bar{y}, 0)\left(\lambda_{i}\right) \text { for } i=1, \ldots, m ; \\
& \left(x_{i}^{*}, y_{i}^{*}\right) \in D^{*} \varphi_{i}(\bar{x}, \bar{y})\left(\lambda_{i}\right) \text { for } i=m+1, \ldots, m+r ; \\
& \left(x_{G}^{*}, y_{G}^{*}\right) \in D^{*} G(\bar{x}, \bar{y}, \bar{w})\left(w^{*}\right) ; \\
& \left(x_{Q}^{*}, y_{Q}^{*}\right) \in D^{*} Q(\bar{x}, \bar{y},-\bar{w})\left(w^{*}\right) \\
& \left(x_{\Omega}^{*}, y_{\Omega}^{*}\right) \in N((\bar{x}, \bar{y}) ; \Omega) ; \\
& \left(x^{*}, y^{*}\right)+\sum_{i=1}^{m+r}\left(x_{i}^{*}, y_{i}^{*}\right)+\left(x_{G}^{*}, y_{G}^{*}\right)+\left(x_{Q}^{*}, y_{Q}^{*}\right)+\left(x_{\Omega}^{*}, y_{\Omega}^{*}\right)=0 .
\end{aligned}
$$

If furthermore $\varphi_{i}$ is upper semicontinuous at $(\bar{x}, \bar{y})$ for each $i=1, \ldots, m$ with $\varphi_{i}(\bar{x}, \bar{y})<0$, then we have in addition the complementary slackness conditions:

$$
\lambda_{i} \varphi_{i}(\vec{x}, \tilde{y})=0 \text { for all } i=1, \ldots, m .
$$

Proof. Consider the product space $\mathbb{R}^{m+r} \times X \times Y \times Z \times W$ endowed with the standard sum norm and construct the following subsets of this space:

$$
\begin{aligned}
& \Omega_{0}:=\mathbb{R}^{m+r} \times \operatorname{gph} f \times W, \\
& \Omega_{i}:=\left\{(\alpha, x, y, z, w) \mid \alpha_{i} \geq \varphi_{i}(x, y)\right\} \text { for } i=1, \ldots, m, \\
& \Omega_{i}:=\left\{(\alpha, x, y, z, w) \mid \alpha_{i}=\varphi_{i}(x, y)\right\} \text { for } i=m+1, \ldots, m+r, \\
& \Omega_{G}:=\{(\alpha, x, y, z, w) \mid(x, y, w) \in \operatorname{gph} G\}, \\
& \Omega_{Q}:=\{(\alpha, x, y, z, w) \mid(x, y,-w) \in \operatorname{gph} Q\}, \\
& \Omega_{\Omega}:=\mathbb{R}_{\leq}^{m} \times 0 \times \Omega \times(\bar{z}+\Theta) \times W,
\end{aligned}
$$

where $\alpha:=\left(\alpha_{1}, \ldots, \alpha_{m+r}\right) \in \mathbb{R}^{m+r}$. It is easy to see that all these sets are locally closed around the point $(0, \vec{x}, \bar{y}, \bar{z}, \bar{w})$. Furthermore, the space $\mathbb{R}^{m+r} \times X \times Y \times Z \times W$ is Asplund as the product of Asplund spaces; see Ref. 25 .

Let us show that the point $(0, \bar{x}, \bar{y}, \bar{z}, \bar{w})$ is locally extremal to the systems of locally closed sets defined in (20). It is easy to see that this point belongs to each of the sets in 
(20). To check the local extremality, we need to justify condition (14) along some sequences $a_{i k} \rightarrow 0$ as $k \rightarrow \infty$. Indeed, by the local $(f, \Theta)$-optimality of the point $(\bar{x}, \bar{y})$ to the MOEC problem (8), there exist a neighborhood $U$ of $(\bar{x}, \bar{y})$ and a sequence $\left\{z_{k}\right\} \subset Z$ with $\left\|z_{k}\right\| \rightarrow 0$ as $k \rightarrow \infty$ satisfying the relationships

$$
f(x, y)-f(\bar{x}, \bar{y}) \notin \Theta-z_{k} \text { for all }(x, y) \in \Xi \cap U \text { for all } k \in \mathbb{N},
$$

where $\Xi$ stands for the set of feasible solutions to (8). To justify the extremal property (14) of the set system $\left\{\Omega_{0}, \Omega_{1}, \ldots, \Omega_{m+r}, \Omega_{G}, \Omega_{Q}, \Omega_{\Omega}\right\}$, it suffices to show that

$$
\bigcap_{i=0}^{m+r} \Omega_{i} \cap \Omega_{G} \cap \Omega_{Q} \cap\left(\Omega_{\Omega}+\left(0, z_{k}, 0\right)\right) \cap V=\emptyset \text { for all } k \in \mathbb{N},
$$

where $V:=\mathbb{R}^{m+r} \times U \times Z \times W$. Arguing by contradiction, assume that this does not hold and so find $(\alpha, x, y, z, w) \in V$ such that

$$
(\alpha, x, y, z, w) \in \bigcap_{i=0}^{m+r} \Omega_{i} \cap \Omega_{G} \cap \Omega_{Q} \cap\left(\Omega_{\Omega}+\left(0, z_{k}, 0\right)\right) .
$$

Hence, by the construction of sets (20), we have the following:

- $(x, y)$ satisfies the inequalities and the equalities in MOEC (8), since

$$
\begin{aligned}
& (\alpha, x, y, z, w) \in \bigcap_{i=1}^{m+r} \Omega_{i} \cap\left(\Omega_{\Omega}+\left(0, z_{k}, 0\right)\right), \\
& \left(x, y, \alpha_{i}\right) \in \operatorname{epi} \varphi_{i}, \text { and } \alpha_{i} \leq 0 \text { for } i=1, \ldots, m, \\
& \left(x, y, \alpha_{i}\right) \in \operatorname{gph} \varphi_{i}, \text { and } \alpha_{i}=0 \text { for } i=m+1, \ldots, m+r, \\
& \varphi_{i}(x, y) \leq \alpha_{i} \leq 0 \text { for } i=1, \ldots, m, \\
& \varphi_{i}(x, y)=\alpha_{i}=0 \text { for } i=m+1, \ldots, m+r
\end{aligned}
$$

- $(x, y)$ satisfies the equilibrium constraint in $\operatorname{MOEC}(8)$, since

$$
\begin{aligned}
& (\alpha, x, y, z, w) \in \Omega_{G} \cap \Omega_{Q} \\
& (x, y, w) \in \operatorname{gph} G \cap \operatorname{gph}(-Q), \\
& w \in G(x, y),-w \in Q(x, y) \\
& 0 \in G(x, y)+Q(x, y)
\end{aligned}
$$

- $(x, y)$ satisfies the geometric constraint in MOEC $(8)$, since $(\alpha, x, y, z, w) \in \Omega_{\Omega}$.

This shows that the pair $(x, y)$ is a feasible solution to the MOEC problem under consideration, i.e., $(x, y) \in \Xi$. Furthermore, we also have from (22) that

$$
\begin{aligned}
& (\alpha, x, y, z, w) \in \Omega_{0} \cap\left(\Omega_{\Omega}+\left(0, z_{k}, 0\right)\right) \\
& (x, y, z) \in \operatorname{gph} f, z \in \Theta-z_{k}, \\
& f(x, y)-f(\bar{x}, \bar{y}) \in \bar{z}+\Theta-z_{k} \text { for all } k \in \mathbb{N}
\end{aligned}
$$

which contradicts the local $(f, \Theta)$-optimality of $(\bar{x}, \bar{y})$ to $(8)$ and so justifies the extremal property (14) of sets (20) at the point $(0, \bar{x}, \bar{y}, \bar{z}, \bar{w})$. 
Thus we can apply to sets $(20)$ at the point $(0, \bar{x}, \bar{y}, \bar{z}, \bar{w})$ the extremal principle formulated in Section 2 (see Theorem 2.22 in Ref. 16) assuming that all but one of these sets are $S N C$ at $(0, \bar{x}, \bar{y}, \bar{z}, \bar{w})$. It is easy to see, by the structures in (20), that the latter SNC properties reduce to the SNC assumptions on the designated sets from (8) at the corresponding points imposed in the theorem. Applying now the extremal principle to sets (20), we conclude from (15), (16), and (20) that there are dual elements

$$
\begin{aligned}
& \left(0, x^{*}, y^{*},-z^{*}, 0\right) \in N\left((0, \bar{x}, \bar{y}, \bar{z}, \bar{w}) ; \Omega_{0}\right), \\
& \left(0,-\lambda_{i}, 0, x_{i}^{*}, y_{i}^{*}, 0\right) \in N\left((0, \bar{x}, \bar{y}, \bar{z}, \bar{w}) ; \Omega_{i}\right), \\
& \left(0, x_{G}^{*}, y_{G}^{*}, 0,-w^{*}\right) \in N\left((0, \bar{x}, \bar{y}, \bar{z}, \bar{w}) ; \Omega_{G}\right), \\
& \left(0, x_{Q}^{*}, y_{Q}^{*}, 0, w^{*}\right) \in N\left((0, \bar{x}, \bar{y}, \bar{z}, \bar{w}) ; \Omega_{Q}\right), \\
& \left(\lambda_{1}, \ldots, \lambda_{m+r}, x_{\Omega}^{*}, y_{\Omega}^{*}, z^{*}, 0\right) \in N\left((0, \bar{x}, \bar{y}, \bar{z}, \bar{w}) ; \Omega_{\Omega}\right),
\end{aligned}
$$

with $i=1, \ldots, m+r$ satisfying the relationships

$$
\begin{aligned}
& \left(x^{*}, y^{*}\right)+\sum_{i=1}^{m+r}\left(x_{i}^{*}, y_{i}^{*}\right)+\left(x_{G}^{*}, y_{G}^{*}\right)+\left(x_{Q}^{*}, y_{Q}^{*}\right)+\left(x_{\Omega}^{*}, y_{\Omega}^{*}\right)=0 \text { and } \\
& \left\|\left(x^{*}, y^{*}\right)\right\|+\sum_{i=1}^{m+r}\left\|\left(x_{i}^{*}, y_{i}^{*}\right)\right\|+\left\|\left(x_{G}^{*}, y_{G}^{*}\right)\right\|+\left\|\left(x_{Q}^{*}, y_{Q}^{*}\right)\right\|+\left\|\left(x_{\Omega}^{*}, y_{\Omega}^{*}\right)\right\| \\
& +2\left(\sum_{i=1}^{m+r}\left|\lambda_{i}\right|+\left\|z^{*}\right\|+\left\|w^{*}\right\|\right)=1 .
\end{aligned}
$$

It is obvious that the last equality implies the nontriviality condition (17) of the theorem.

To derive the necessary optimality conditions (18) from the above relationships, we exploit the structures of the sets in (20) together with the definitions of coderivatives and subgradients given in Section 2 and the product formula for computing basic normals:

$$
N\left(\left(\bar{x}_{1}, \bar{x}_{2}\right) ; \Omega_{1} \cap \Omega_{2}\right)=N\left(\bar{x}_{1} ; \Omega_{1}\right) \times N\left(\bar{x}_{2} ; \Omega_{2}\right) .
$$

In this way we get the following:

- (23) implies $\left(x^{*}, y^{*}\right) \in D^{*} f(\bar{x}, \bar{y})\left(z^{*}\right)$;

- (24) and (25) imply

$$
\begin{aligned}
&\left(x_{i}^{*}, y_{i}^{*}\right) \in D^{*} E_{\varphi_{i}}(\bar{x}, \bar{y}, 0)\left(\lambda_{i}\right) \text { for } i=1, \ldots, m, \\
&\left(x_{i}^{*}, y_{i}^{*}\right) \in D^{*} \varphi_{i}(\bar{x}, \bar{y})\left(\lambda_{i}\right) \text { for } i=m+1, \ldots, m+r, \\
&\left(x_{G}^{*}, y_{G}^{*}\right) \in D^{*} G(\bar{x}, \bar{y}, \bar{w})\left(w^{*}\right) ; \\
& \cdot\left(x_{Q}^{*}, y_{Q}^{*}, w^{*}\right) \in N((\bar{x}, \bar{y}, \bar{w}) ; \operatorname{gph}(-Q)), \\
&\left(x_{Q}^{*}, y_{Q}^{*},-w^{*}\right) \in N((\bar{x}, \bar{y},-\bar{w}) ; \operatorname{gph} Q), \\
&\left(x_{Q}^{*}, y_{Q}^{*}\right) \in D^{*} Q(\bar{x}, \bar{y},-\bar{w})\left(w^{*}\right) . \\
& \\
&\left(\lambda_{1}, \ldots, \lambda_{m}\right) \in N\left(0 ; \mathbb{R}_{\leq}^{m}\right)=\mathbb{R}_{\geq}^{m}, \text { i.e., } \\
& \lambda_{1}, \ldots, \lambda_{m} \geq 0, \\
&\left(\lambda_{m+1}, \ldots, \lambda_{m+r}\right) \in N(0 ; 0)=\mathbb{R}^{r}, \\
&\left(x_{\Omega}^{*}, y_{\Omega}^{*}\right) \in N((\bar{x}, \bar{y}) ; \Omega), \\
& z^{*} \in N(\bar{z} ;(\bar{z}+\Theta))=N(0 ; \Theta) .
\end{aligned}
$$$$
\text { - (27) gives } \quad\left(\lambda_{1}, \ldots, \lambda_{m}\right) \in N\left(0 ; \mathbb{R}_{\leq}^{m}\right)=\mathbb{R}_{\geq}^{m} \text {, i.e., }
$$ 
It remains to justify that the complementary slackness conditions (19) hold provided that the functions $\varphi_{i}$ are upper semicontinuous at $(\bar{x}, \bar{y})$ for $i \in\{1, \ldots, m\}$ with $\varphi_{i}(\bar{x}, \bar{y})<0$. Indeed, the latter assumption imposed for each such index $i$ implies in fact that $(\vec{x}, \vec{y}, 0)$ is an interior point of the epigraph of the corresponding function $\varphi_{i}$. Thus

$$
N\left((\bar{x}, \bar{y}, 0) ; \operatorname{epi} \varphi_{i}\right)=\{0\}
$$

and $x_{i}^{*}=y_{i}^{*}=\lambda_{i}=0$ for this $i$, which completes the proof of the theorem.

The next theorem provides an improvement of necessary optimality conditions of Theorem 3.2 under additional assumptions. To establish this theorem, we need to recall the notion of the mixed coderivative for a set-valued mapping $F: X \rightrightarrows Y$ between Banach (in fact Asplund) spaces at $(\bar{x}, \bar{y}) \in \operatorname{gph} F$ defined as follows:

$$
\begin{aligned}
& D_{M}^{*} F(\bar{x}, \bar{y})\left(y^{*}\right):=\left\{x^{*} \in X^{*} \mid \exists \text { sequences }\left(x_{k}, y_{k}\right) \rightarrow(\bar{x}, \bar{y}), x_{k}^{*} \stackrel{w^{*}}{\rightarrow} x^{*}, y_{k}^{*} \rightarrow y^{*}\right. \\
& \text { with } \left.\left(x_{k}, y_{k}\right) \in \operatorname{gph} F,\left(x_{k}^{*},-y_{k}^{*}\right) \in \widehat{N}\left(\left(x_{k}, y_{k}\right) ; \operatorname{gph} F\right)\right\} \text {. }
\end{aligned}
$$

Comparing this construction with that (11) for the normal coderivative and taking into account definitions (9) and (10) of the basic and Fréchet normal cones, we observe that the only difference between $D^{*} F(\bar{x}, \bar{y})\left(y^{*}\right)$ and $D_{M}^{*} F(\bar{x}, \bar{y})\left(y^{*}\right)$ consists of using the norm convergence $\left\|y_{k}^{*}-y^{*}\right\| \rightarrow 0$ for the mixed coderivative instead of the weak* convergence $y_{k}^{*} \stackrel{w^{*}}{\rightarrow} y^{*}$ for the normal one, while the weak ${ }^{*}$ convergence $x_{k}^{*} \stackrel{w^{*}}{\rightarrow} x^{*}$ is used in both cases. We obviously have

$$
D_{M}^{*} F(\bar{x}, \bar{y})\left(y^{*}\right) \subset D^{*} F(\bar{x}, \bar{y})\left(y^{*}\right) \text { for all } y^{*} \in Y^{*},
$$

where the equality holds if $Y$ is finite-dimensional. In fact, the equality holds in (28) in broad classes of mappings with infinite-dimensional images; the latter property is postulated in Ref. 16 as strong coderivative normality of the mapping $F$ at the point $(\bar{x}, \bar{y})$. We refer the reader to Proposition 4.9 in Ref. 16, which summarizes major classes of single-valued and set-valued mappings that are strongly coderivatively normal and presents also some calculus rules for this important property.

A significant advantage of the mixed coderivative is that

$$
D_{M}^{*} F(\bar{x}, \bar{y})(0)=\{0\}
$$

provided that $F$ enjoys a certain robust Lipschitzian behavior around the points in question. Namely, (29) always holds if a locally closed-graph mapping $F: X \rightrightarrows Y$ is Lipschitz-like around $(\bar{x}, \bar{y})$ with modulus $\ell \geq 0$, i.e., there are neighborhoods $U$ of $\bar{x}$ and $V$ of $\bar{y}$ such that

$$
F(x) \cap V \subset F(u)+\ell\|x-u\| \mathbb{B} \text { for all } x, u \in U,
$$

where $\mathbb{B}$ stands for the closed unit ball of $Y$. This property, which is also known as the Aubin or pseudo-Lipschitzian property (cf. the discussions in Refs. 16 and 23), agrees with the classical local Lipschitzian behavior in the case of single-valued mappings and reduces to the standard (Hausdorff) local Lipschitzian property of set-valued mappings when $V=Y$. Furthermore, it is equivalent to both metric regularity and linear openness properties of the inverse mapping $F^{-1}$; see the afore-mentioned books and their references. It happens that the coderivative condition (29) is not only necessary for the Lipschitz-like property of $F$ around $(\bar{x}, \vec{y})$ in arbitrary Banach spaces but also sufficient for this property in the 
framework of Asplund spaces under a certain "partial SNC" assumption that is automatic if the range space $Y$ is of finite dimension; see Theorem 4.10 in Ref. 16 for the full account of this coderivative criterion and also Theorem 7.40 in Ref. 23 with the references therein for the case of mappings between finite-dimensional spaces.

Theorem 3.3 (necessary conditions for general MOECs with enhanced nontriviality). Given a local $(f, \Theta)$-optimal solution $(\widetilde{x}, \bar{y})$ to the MOEC problem (8), assume in addition to the hypotheses of Theorem 3.2 that $\Theta$ is SNC at 0 and that the following qualification condition is fulfilled: one has

$$
\begin{aligned}
& {\left[\left(x^{*}, y^{*}\right)+\sum_{i=1}^{m+r}\left(x_{i}^{*}, y_{i}^{*}\right)+\left(x_{G}^{*}, y_{G}^{*}\right)+\left(x_{Q}^{*}, y_{Q}^{*}\right)+\left(x_{\Omega}^{*}, y_{\Omega}^{*}\right)=0\right]} \\
& \Longrightarrow\left(x^{*}, y^{*}\right)=\left(x_{i}^{*}, y_{i}^{*}\right)=\left(x_{G}^{*}, y_{G}^{*}\right)=\left(x_{Q}^{*}, y_{Q}^{*}\right)=\left(x_{\Omega}^{*}, y_{\Omega}^{*}\right)=0 \\
& \text { whenever }\left(x^{*}, y^{*}\right) \in D_{M}^{*} f(\bar{x}, \bar{y})(0), \\
& \left(x_{i}^{*}, y_{i}^{*}\right) \in \partial^{\infty} \varphi_{i}(\bar{x}, \bar{y}) \text { for } i=1, \ldots, m, \\
& \left(x_{i}^{*}, y_{i}^{*}\right) \in \partial^{\infty} \varphi_{i}(\bar{x}, \bar{y}) \cup \partial^{\infty}\left(-\varphi_{i}\right)(\bar{x}, \bar{y}) \text { for } i=m+1, \ldots, m+r, \\
& \left(x_{G}^{*}, y_{G}^{*}\right) \in D^{*} G(\bar{x}, \bar{y}, \bar{w})(0) \\
& \left(x_{Q}^{*}, y_{Q}^{*}\right) \in D^{*} Q(\bar{x}, \bar{y},-\bar{w})(0), \\
& \left(x_{\Omega}^{*}, y_{\Omega}^{*}\right) \in N((\bar{x}, \bar{y}) ; \Omega)
\end{aligned}
$$

the latter qualification requirement automatically holds if the mapping $f$ and functions $\varphi_{i}$ as $i=1, \ldots, m+r$ are locally Lipschitzian around $(\bar{x}, \bar{y})$ while the set-valued mappings $G$ and $Q$ are Lipschitz-like around $(\bar{x}, \bar{y}, \bar{w})$ and $(\bar{x}, \bar{y},-\bar{w})$ and strongly coderivatively normal at the corresponding points. Then we can choose dual elements

$$
\lambda_{1}, \ldots, \lambda_{m+r}, z^{*}, w^{*}, x^{*}, y^{*}, x_{1}^{*}, y_{1}^{*}, \ldots, x_{m+r}^{*}, y_{m+r}^{*}, x_{G}^{*}, y_{G}^{*}, x_{Q}^{*}, y_{Q}^{*}, x_{\Omega}^{*}, y_{\Omega}^{*}
$$

satisfying the relationships of Theorem 3.2 and the enhanced nontriviality condition:

$$
\left(\lambda_{1}, \ldots, \lambda_{m+r}, z^{*}, w^{*}\right) \neq 0 .
$$

Proof. As shown in the proof of Theorem 3.2, the point $(0, \vec{x}, \bar{y}, \bar{z}, \bar{w})$ is locally extremal for the system of locally closed sets (20) in the Asplund space $\mathbb{R}^{m+r} \times X \times Y \times Z \times W$. Applying to this set system the approximate version of the extremal principle from Theorem 2.20 of Ref. 16 , we find sequences

$$
\begin{aligned}
& \left(0, x_{k}^{*}, y_{k}^{*},-z_{k}^{*}, 0\right) \in \widehat{N}\left(\left(\alpha_{k}, x_{k}, y_{k}, z_{k}, w_{k}\right) ; \Omega_{0}\right), \\
& \left(0,-\lambda_{i k}, 0, x_{i k}^{*}, y_{i k}^{*}, 0\right) \in \widehat{N}\left(\left(\alpha_{i k}, x_{i k}, y_{i k}, z_{i k}, w_{i k}\right) ; \Omega_{i}\right), \\
& \left(0, x_{G k}^{*}, y_{G k}^{*}, 0,-w_{G k}^{*}\right) \in \widehat{N}\left(\left(\alpha_{G k}, x_{G k}, y_{G k}, z_{G k}, w_{G k}\right) ; \Omega_{G}\right), \\
& \left(0, x_{Q k}^{*}, y_{Q k}^{*}, 0, w_{Q k}^{*}\right) \in \widehat{N}\left(\left(\alpha_{Q k}, x_{Q k}, y_{Q k}, z_{Q k}, w_{Q k}\right) ; \Omega_{Q}\right), \\
& \left(\lambda_{k}^{1}, \ldots, \lambda_{k}^{m+r}, x_{\Omega k}^{*}, y_{\Omega k}^{*}, z_{\Omega k}^{*}, 0\right) \in \widehat{N}\left(\left(\alpha_{\Omega k}, x_{\Omega k}, y_{\Omega k}, z_{\Omega k}, w_{\Omega k}\right) ; \Omega_{\Omega}\right)
\end{aligned}
$$

with $i=1, \ldots, m+r$ satisfying the following relationships:

$$
\begin{aligned}
& \left(x_{i k}, y_{i k}, z_{i k}, w_{i k}\right) \rightarrow(\bar{x}, \bar{y}, \bar{z}, \bar{w}), \quad\left(\alpha_{k}, \alpha_{G k}, \alpha_{Q k}, \alpha_{\Omega k}\right) \rightarrow(0,0,0,0), \\
& \alpha_{i k} \rightarrow 0, \quad\left|-\lambda_{i k}+\lambda_{k}^{i}\right| \rightarrow 0 \text { for } i=1, \ldots, m+r, \\
& \left\|-z_{k}^{*}+z_{\Omega k}^{*}\right\| \rightarrow 0, \quad\left\|-w_{G k}^{*}+w_{Q k}^{*}\right\| \rightarrow 0, \quad \text { and } \\
& \left\|\left(x_{k}^{*}, y_{k}^{*}\right)+\sum_{i=1}^{m+r}\left(x_{i k}^{*}, y_{i k}^{*}\right)+\left(x_{G k}^{*}, y_{G k}^{*}\right)+\left(x_{Q k}^{*}, y_{Q k}^{*}\right)+\left(x_{\Omega k}^{*}, y_{\Omega k}^{*}\right)\right\| \rightarrow 0
\end{aligned}
$$


as $k \rightarrow \infty$, where the sequences of dual elements are uniformly bounded. Since they belong to duals to Asplund spaces, we may assume without loss of generality (see Ref. 25) that all of them weak ${ }^{*}$ sequentially converge to the corresponding limiting elements, which thus satisfy the necessary optimality conditions of Theorem 3.2. Let us justify the enhanced nontriviality condition (31) under the additional assumptions made above.

Arguing by contradiction, suppose that

$$
\left(\lambda_{1}, \ldots, \lambda_{m+r}, z^{*}, w^{*}\right)=0 .
$$

Then $z_{\Omega k}^{*} \stackrel{w^{*}}{\longrightarrow} 0$ with $z_{\Omega k}^{*} \in \widehat{N}\left(z_{\Omega k} ;(\ddot{z}+\Theta)\right)$ due to the structure of the set $\Omega_{\Omega}$ in $(20)$. Since $\Theta$ is SNC at 0 , we have

$$
\left\|z_{\Omega k}^{*}\right\| \rightarrow 0 \text { and thus }\left\|z_{k}^{*}\right\| \rightarrow 0
$$

by $\left\|-z_{k}^{*}+z_{\Omega k}^{*}\right\| \rightarrow 0$ as $k \rightarrow \infty$ in (32). This implies, by the definition of the mixed coderivative, that $\left(x^{*}, y^{*}\right) \in D_{M}^{*} f(\bar{x}, \bar{y})(0)$. Using further (18) with $\lambda_{i}=0$ and the representation of the coderivative in (13), we have

$$
\begin{aligned}
& \left(x_{i}^{*}, y_{i}^{*}\right) \in \partial^{\infty} \varphi_{i}(\bar{x}, \bar{y}) \text { for } i=1, \ldots, m, \text { and } \\
& \left(x_{i}^{*}, y_{i}^{*}\right) \in \partial^{\infty} \varphi_{i}(\bar{x}, \bar{y}) \cup \partial^{\infty}\left(-\varphi_{i}\right)(\bar{x}, \bar{y}) \text { for } i=m+1, \ldots, m+r .
\end{aligned}
$$

Then the qualification assumption (30) yields that

$$
\left(x^{*}, y^{*}\right)=\left(x_{i}^{*}, y_{i}^{*}\right)=\left(x_{G}^{*}, y_{G}^{*}\right)=\left(x_{Q}^{*}, y_{Q}^{*}\right)=\left(x_{\Omega}^{*}, y_{\Omega}^{*}\right)=0,
$$

which contradicts the nontriviality condition (17) of Theorem 3.2 and thus justifies (31). To complete the proof of the theorem, it remains to observe that the fulfilment of (30) under the additional Lipschitzian and coderivative normality assumptions made directly follows from the corresponding definitions and the coderivative criterion (29).

It is worth mentioning the following efficient specification and simplification of both the assumptions and conclusions of Theorem 3.3. Given $\varphi: X \rightarrow \overline{\mathbb{R}}$ finite at $\bar{x}$, we define the symmetric subdifferential of $\varphi$ at $\bar{x}$ by

$$
\partial^{0} \varphi(\bar{x}):=\partial \varphi(\bar{x}) \cup(-\partial(-\varphi)(\bar{x})) .
$$

Corollary 3.4 (necessary optimality conditions for MOECs under Lipschitzian assumptions). Let $(\bar{x}, \bar{y})$ be a local $(f, \Theta)$-optimal solution to the MOEC problem (8), where the spaces $X$ and $Y$ are Asplund while $Z$ and $W$ are finite-dimensional, and where the sets $\Omega \subset X \times Y$ and $\Theta \subset Z$ are locally closed around $(\bar{x}, \bar{y})$ and 0 , respectively. Assume also that the mapping $f: X \times Y \rightarrow Z$ and the functions $\varphi_{i}: X \times Y \rightarrow \mathbb{R}$ as $i=1, \ldots, m+r$ are locally Lipschitzian around $(\bar{x}, \bar{y})$ and that the set-valued mappings $G: X \times Y \rightrightarrows W$ and $Q: X \times Y \rightrightarrows W$ are Lipschitz-like around $(\bar{x}, \bar{y}, \bar{w})$ and $(\bar{x}, \bar{y},-\bar{w})$, respectively, with some given $\bar{w} \in G(\bar{x}, \bar{y}) \cap(-Q(\vec{x}, \bar{y}))$. Then there are

$$
\left(\lambda_{1}, \ldots, \lambda_{m+r}, z^{*}, w^{*}\right) \neq 0 \text { with } z^{*} \in N(0 ; \Theta) \text { and } \lambda_{i} \geq 0 \text { for } i=1, \ldots, m
$$

satisfying the complementary slackness conditions (19) and such that

$$
\begin{aligned}
& 0 \in \partial\left\langle z^{*}, f\right\rangle(\bar{x}, \bar{y})+\sum_{i=1}^{m} \lambda_{i} \partial \varphi_{i}(\bar{x}, \bar{y})+\sum_{i=m+1}^{m+r} \lambda_{i} \partial^{0} \varphi_{i}(\bar{x}, \bar{y}) \\
& +D^{*} G(\bar{x}, \bar{y}, \bar{w})\left(w^{*}\right)+D^{*} Q(\bar{x}, \bar{y},-\bar{w})\left(w^{*}\right)+N((\bar{x}, \bar{y}) ; \Omega) .
\end{aligned}
$$


Proof. Let us first check that all the assumptions of Theorem 3.3 (and those from Theorem 3.2) are satisfied under the assumptions made in this corollary. Since the SNC property of $\Theta$ and the strong coderivative normality property of $G$ and $Q$ are obvious due to the finite dimensionality of the spaces $Z$ and $W$, we need to justify the fulfillment of the SNC property of the epigraphical sets epi $\varphi_{i}$ for $i=1, \ldots, m$ and the graphical sets gph $\varphi_{i}$ for $i=m+1, \ldots, m+r$ and $\operatorname{gph} f$ when $\varphi_{i}$ and $f$ are locally Lipschitzian around $(\bar{x}, \bar{y})$, as well as the fulfillment of the SNC property of $\operatorname{gph} G$ and $\operatorname{gph}(-Q)$ at the corresponding points under the Lipschitz-like requirements imposed on $G$ and $Q$. All these SNC conclusions follow from Proposition 1.68 and Corollary 1.69 of Ref. 16 by the finite dimensionality of the spaces $Z$ and $W$. Furthermore, we have the scalarization formula

$$
D^{*} f(\vec{x}, \tilde{y})\left(z^{*}\right)=\partial\left\langle z^{*}, f\right\rangle(\bar{x}, \bar{y}) \text { for all } z^{*} \in Z^{*}
$$

due to the Lipschitz continuity of $f$ around $(\bar{x}, \bar{y})$ and $\operatorname{dim} Z<\infty$; see Theorem 1.90 from Ref. 16. It is easy to observe the relationships

$$
D^{*} E_{\varphi}(\bar{t}, \varphi(\bar{t}))(\lambda)=\lambda \partial \varphi(\bar{t}) \text { with } \lambda \geq 0 \text { and } D^{*} \varphi(\bar{t})(\lambda) \subset \lambda \partial^{0} \varphi(\bar{t}) \text { with } \lambda \in \mathbb{R}
$$

held for any function $\varphi: T \rightarrow \bar{R}$ locally Lipschitzian around $\bar{t}$. Taking all these into account, we arrive at (33) from the optimality conditions (18) of Theorem 3.2.

Remark 3.5 (multiobjective optimization problems with respect to closed preferences). The results obtained in this section establish necessary optimality conditions for general MOEC problems (8), where the notion of multiobjective optimality is understood in the sense of Definition 3.1. Following the above arguments and the corresponding developments in Subsection 5.3.5 of Ref. 7, we can also derive necessary optimality conditions for general MOECs of type (8) and their specifications considered below for multiobjective problems with equilibrium constraints, where the notion of vector optimality is defined by certain closed preference relations. Note that the latter concept of multiobjective optimization is generally different from that of Definition 3.1; see Refs. 7 and 29 for more discussions, examples, and applications.

\section{MOECs with Finitely Many Objectives}

In this section we establish certain versions of necessary optimality conditions from Section 3 for the case of MOECs with finitely many objectives and derive their specifications for general problems of multiobjective fractional programming with equilibrium constraints.

Given $f_{j}: X \times Y \rightarrow \widetilde{\mathbb{R}}$ as $j=1, \ldots, n$, consider the following multiobjective problem:

$$
\begin{array}{ll}
\text { minimize } & f(x, y)=\left(f_{1}(x, y), \ldots, f_{n}(x, y)\right) \\
\text { subject to } & \varphi_{i}(x, y) \leq 0, \quad i=1, \ldots, m, \\
& \varphi_{i}(x, y)=0, \quad i=m+1, \ldots, m+r, \\
& (x, y) \in \Omega \\
& 0 \in G(x, y)+Q(x, y),
\end{array}
$$

where the vector "minimization" is understood in the sense of Definition 3.1 with $\Theta=R_{\leq}^{n}$ (i.e., in the sense of (weak) Pareto) and where the other data are the same as in the general constraint MOEC problem (8).

The next result presents major necessary optimality conditions for (34), which are in the line of Theorem 3.3. Observe, however, that the assumptions of the following theorem are 
less restrictive in comparison with Theorem 3.3 due to the nature of the objective mapping $f$ in (34), where the component functions $f_{j}$ can be assumed to be merely lower semicontinuous (vs. full continuity in Theorem 3.3) around the local minimizer. Furthermore, some conclusions of this theorem are stronger than those of Theorem 3.3; see the discussion and example presented after the proof of the theorem.

Theorem 4.1 (necessary optimality conditions for general MOECs with finitely many objectives). Let $(\bar{x}, \bar{y})$ be a local optimal solution to problem (34), where the spaces $X, Y$, and $W$ are Asplund. Given $\bar{w} \in G(\bar{x}, \bar{y}) \cap \operatorname{gph}(-Q)$ and $\bar{z}_{j}:=f_{j}(\vec{x}, \bar{y})$, assume that the functions $f_{j}$ as $j=1, \ldots, n$ and $\varphi_{i}$ as $i=1, \ldots, m$ are l.s.c. around $(\bar{x}, \bar{y})$ while $\varphi_{i}$ are continuous around this point for $i=m+1, \ldots, m+r$. Suppose also that the all but one of the sets epi $f_{j}$ as $j=1, \ldots, n$, epi $\varphi_{i}$ as $i=1, \ldots, m, \operatorname{gph} \varphi_{i}$ as $i=m+1, \ldots, m+r, \operatorname{gph} G$, $\operatorname{gph} Q$, and $\Omega$ are $S N C$ at $\left(\bar{x}, \bar{y}, \bar{z}_{j}\right),(\bar{x}, \bar{y}, 0),(\bar{x}, \bar{y}, \bar{w}),(\bar{x}, \bar{y},-\bar{w})$, and $(\bar{x}, \bar{y})$, respectively. Furthermore, we imposed the following qualification condition: one has

$$
\begin{aligned}
& {\left[\sum_{j=1}^{n}\left(\xi_{j}^{*}, \zeta_{j}^{*}\right)+\sum_{i=1}^{m+r}\left(x_{i}^{*}, y_{i}^{*}\right)+\left(x_{G}^{*}, y_{G}^{*}\right)+\left(x_{Q}^{*}, y_{Q}^{*}\right)+\left(x_{\Omega}^{*}, y_{\Omega}^{*}\right)=0\right]} \\
& \Longrightarrow\left(\xi_{j}^{*}, \zeta_{j}^{*}\right)=\left(x_{i}^{*}, y_{i}^{*}\right)=\left(x_{G}^{*}, y_{G}^{*}\right)=\left(x_{Q}^{*}, y_{Q}^{*}\right)=\left(x_{\Omega}^{*}, y_{\Omega}^{*}\right)=0
\end{aligned}
$$

provided that $\left(\xi_{j}^{*}, \zeta_{j}^{*}\right) \in \partial^{\infty} f_{j}(\bar{x}, \bar{y})$ as $j=1, \ldots, n$ and that $x_{i}^{*}, y_{i}^{*}, x_{G}^{*}, y_{G}^{*}, x_{Q}^{*}, y_{Q}^{*}, x_{\Omega}^{*}, y_{\Omega}^{*}$ are the same as in the qualification condition (30); note that (35) is automatic under the Lipschitzian and coderivative normality assumptions mentioned in Theorem 3.3. Then there exist dual elements

$$
\begin{aligned}
& \nu_{j} \geq 0 \text { for } j=1, \ldots, n, w^{*} \in W^{*}, \\
& \lambda_{i} \geq 0 \text { for } i=1, \ldots, m, \lambda_{i} \in \mathbb{R} \text { for } i=m+1, \ldots, m+r, \\
& \left(\xi_{j}^{*}, \zeta_{j}^{*}\right) \in D^{*} E_{f_{j}}\left(\bar{x}, \bar{y}, \bar{z}_{j}\right)\left(\nu_{j}\right) \text { for } j=1, \ldots, n, \\
& \left(x_{i}^{*}, y_{i}^{*}\right) \in D^{*} E_{\varphi_{i}}(\bar{x}, \bar{y}, 0)\left(\lambda_{i}\right) \text { for } i=1, \ldots, m, \\
& \left(x_{i}^{*}, y_{i}^{*}\right) \in D^{*} \varphi_{i}(\bar{x}, \bar{y})\left(\lambda_{i}\right) \text { for } i=m+1, \ldots, m+r, \\
& \left(x_{G}^{*}, y_{G}^{*}\right) \in D^{*} G(\bar{x}, \bar{y}, \bar{w})\left(w^{*}\right), \\
& \left(x_{Q}^{*}, y_{Q}^{*}\right) \in D^{*} Q(\bar{x}, \bar{y},-\bar{w})\left(w^{*}\right), \\
& \left(x_{\Omega}^{*}, y_{\Omega}^{*}\right) \in N((\bar{x}, \bar{y}) ; \Omega)
\end{aligned}
$$

satisfying the complementary slackness conditions (19), the generalized Euler equation

$$
\sum_{j=1}^{n}\left(\xi_{j}^{*}, \zeta_{j}^{*}\right)+\sum_{i=1}^{m+r}\left(x_{i}^{*}, y_{i}^{*}\right)+\left(x_{G}^{*}, y_{G}^{*}\right)+\left(x_{Q}^{*}, y_{Q}^{*}\right)+\left(x_{\Omega}^{*}, y_{\Omega}^{*}\right)=0
$$

and the enhanced nontriviality condition

$$
\left(\nu_{1}, \ldots, \nu_{n}, \lambda_{1}, \ldots, \lambda_{m+r}, w^{*}\right) \neq 0 .
$$

Proof. We proceed similarly to the proof of Theorem 3.2 and then that of Theorem 3.3 with the replacement of the extremal system (20) by the new one

$$
\left\{\mathcal{O}_{1}, \ldots, \mathcal{O}_{n}, \Omega_{1}, \ldots, \Omega_{m+r}, \Omega_{G}, \Omega_{Q}, \Omega_{\Omega}\right\}
$$


where the sets $\mathcal{O}_{j}$ and $\Omega_{\Omega}$ are defined by

$$
\begin{aligned}
& \mathcal{O}_{j}:=\left\{(\alpha, x, y, z, w) \mid z_{j} \geq f_{j}(\bar{x}, \bar{y})\right\}, \quad j=1, \ldots, n, \\
& \Omega_{\Omega}:=\mathbb{R}_{\leq}^{m} \times 0 \times \Omega \times\left(\bar{z}+\mathbb{R}_{\leq}^{n}\right) \times W
\end{aligned}
$$

while $\Omega_{i}, \Omega_{G}$, and $\Omega_{Q}$ are the same as in (20). It is easy to check that

$$
\left(0, \xi_{j}^{*}, \zeta_{j}^{*}, 0, \nu_{j}, 0\right) \in N\left((0, \bar{x}, \bar{y}, \bar{z}, \bar{w}) ; \mathcal{O}_{j}\right) \Longrightarrow\left[\nu_{j} \geq 0,\left(\xi_{j}^{*}, \zeta_{j}^{*}\right) \in D^{*} E_{f_{j}}(\bar{x}, \bar{y})\left(\nu_{j}\right)\right]
$$

for all $j=1, \ldots, n$. Thus we arrive at all the conclusions of the theorem similarly to the afore-mentioned proofs in Section 3.

Observe that, even for continuous (but not-Lipschitzian) functions $f_{j}$, the conclusion

$$
\left(\xi_{j}^{*}, \zeta_{j}^{*}\right) \in D^{*} E_{f_{j}}\left(\bar{x}, \bar{y}, \bar{z}_{j}\right)\left(\nu_{j}\right), \quad j=1, \ldots, n,
$$

of Theorem 4.1 is stronger (more selective) than the corresponding conclusion

$$
\left(x^{*}, y^{*}\right) \in D^{*} f(\bar{x}, \bar{y})\left(z^{*}\right) \text { with } f=\left(f_{1}, \ldots, f_{n}\right), z^{*}=\left(\nu_{1}, \ldots, \nu_{n}\right)
$$

in (18). This follows from the relationships

$$
D^{*} E_{f_{j}}\left(\bar{x}, \bar{y}, \bar{z}_{j}\right)(0) \subset D^{*} f_{j}(\bar{x}, \bar{y})(0)=\partial^{\infty} f_{j}(\bar{x}, \bar{y}),
$$

where the inclusion is generally strict as, e.g., in the case of

$$
f(x)=\left\{\begin{array}{lll}
-\sqrt[3]{x} & \text { if } & x \geq 0 \\
0 & \text { if } & x<0
\end{array}\right.
$$

with $\partial^{\infty} f(0)=(-\infty, 0]$ and $D^{*} f(0)(0)=(-\infty, \infty)$.

Next we consider the multiobjective fractional program with equilibrium constraints formulated as follows:

$$
\begin{aligned}
& \text { minimize } f(x, y)=\left(\frac{g_{1}(x, y)}{h_{1}(x, y)}, \ldots, \frac{g_{n}(x, y)}{h_{n}(x, y)}\right) \\
& \text { subject to } \varphi_{i}(x, y) \leq 0, \quad i=1, \ldots, m \\
& \varphi_{i}(x, y)=0, \quad i=m+1, \ldots, m+r \\
& (x, y) \in \Omega \\
& 0 \in G(x, y)+Q(x, y)
\end{aligned}
$$

where $g_{j}, h_{j}: X \times Y \rightarrow \bar{R}, j=1, \ldots, n$, are extended-real-valued functions such that $h_{j}(x, y) \neq 0$ at the corresponding points, and where the other data are the same as in (34). The multiobjective minimization in (36) is understood in the same Pareto sense as in (34). Thus (36) is a special case of (34) with

$$
f_{j}(x, y):=\frac{g_{j}(x, y)}{h_{j}(x, y)}, \quad j=1, \ldots, n .
$$

The following theorem, proving necessary optimality conditions for the multiobjective fractional programming problem (36), is a consequence of Theorem 4.1 and the quotient rule of generalized differentiation from Ref. 16. 
Theorem 4.2 (necessary conditions for multiobjective fractional MPECs). Let $(\bar{x}, \bar{y})$ be a local optimal solution to the multiobjective fractional problem (36), where the functions $g_{j}, h_{j}, j=1, \ldots, n$, are Lipschitz continuous around $(\vec{x}, \bar{y})$ with $h_{j}(\bar{x}, \bar{y}) \neq 0$, and where the other data are the assumptions of Theorem 4.1 with the qualification condition formulated now as: for any elements

$$
\begin{aligned}
& \left(x_{i}^{*}, y_{i}^{*}\right) \in \partial^{\infty} \varphi_{i}(\bar{x}, \bar{y}), \quad i=1, \ldots, m, \\
& \left(x_{i}^{*}, y_{i}^{*}\right) \in \partial^{\infty} \varphi_{i}(\bar{x}, \bar{y}) \cup \partial^{\infty}\left(-\varphi_{i}\right)(\bar{x}, \bar{y}), \quad i=m+1, \ldots, m+r \\
& \left(x_{G}^{*}, y_{G}^{*}\right) \in D^{*} G(\bar{x}, \bar{y}, \bar{w})(0) \\
& \left(x_{Q}^{*}, y_{Q}^{*}\right) \in D^{*} Q(\bar{x}, \bar{y},-\bar{w})(0) \\
& \left(x_{\Omega}^{*}, y_{\Omega}^{*}\right) \in N((\bar{x}, \bar{y}) ; \Omega)
\end{aligned}
$$

one has the implication

$$
\begin{aligned}
& {\left[\sum_{i=1}^{m+r}\left(x_{i}^{*}, y_{i}^{*}\right)+\left(x_{G}^{*}, y_{G}^{*}\right)+\left(x_{Q}^{*}, y_{Q}^{*}\right)+\left(x_{\Omega}^{*}, y_{\Omega}^{*}\right)=0\right]} \\
& \Longrightarrow\left(x_{i}^{*}, y_{i}^{*}\right)=\left(x_{G}^{*}, y_{G}^{*}\right)=\left(x_{Q}^{*}, y_{Q}^{*}\right)=\left(x_{\Omega}^{*}, y_{\Omega}^{*}\right)=0 .
\end{aligned}
$$

Then the conclusions of Theorem 4.1 hold with

$$
\left(\xi_{j}^{*}, \zeta_{j}^{*}\right) \in \nu_{j}\left[\frac{\partial\left(h_{j}(\bar{x}, \bar{y}) g\right)(\bar{x}, \bar{y})-\partial\left(g_{j}(\bar{x}, \bar{y}) h\right)(\bar{x}, \bar{y})}{\left[h_{j}(\bar{x}, \bar{y})\right]^{2}}\right]
$$

for some $\nu_{j} \geq 0$ as $j=1, \ldots, n$.

Proof. We need elaborate the necessary conditions

$$
\left(\xi_{j}^{*}, \zeta_{j}^{*}\right) \in D^{*} E_{f_{j}}\left(\bar{x}, \bar{y}, \bar{z}_{j}\right)\left(\nu_{j}\right), \quad j=1, \ldots, n,
$$

of Theorem 4.1 with $f_{j}$ given in $(37)$ and $\bar{z}_{j}:=g_{j}(\bar{x}, \bar{y}) / h_{j}(\bar{x}, \bar{y})$. Since $g_{j}$ and $h_{j}$ are assumed to be locally Lipschitzian around $(\bar{x}, \vec{y})$, the latter conditions reduce to

$$
\left(\xi_{j}^{*}, \zeta_{j}^{*}\right) \in \nu_{j} \partial\left(\frac{g_{j}}{h_{j}}\right)(\bar{x}, \bar{y}), \quad j=1, \ldots, n .
$$

Employing the quotient rule for basic subgradients from Proposition 3.45 in Ref. 16, we get

$$
\partial\left(\frac{g_{j}}{h_{j}}\right)(\bar{x}, \bar{y}) \subset\left[\frac{\partial\left(h_{j}(\bar{x}, \bar{y}) g_{j}\right)(\bar{x}, \bar{y})-\partial\left(g_{j}(\bar{x}, \bar{y}) h_{j}\right)(\bar{x}, \bar{y})}{\left[h_{j}(\bar{x}, \bar{y})\right]^{2}}\right], \quad j=1, \ldots, n,
$$

and thus complete the proof of the theorem.

The results obtained in Theorem 4.2 related to those from Ref. 30, where the authors consider nonsmooth multiobjective fractional programs in finite dimensions with Lipschitzian data and no equilibrium constraints and use Clarke's generalized gradients to formulate necessary (sub)optimality conditions. Note that most of the work on optimality conditions in fractional programming has been carried out under certain assumptions involving convexity and/or invexity; see Ref. 30 and the bibliography therein. 


\section{Multiobjective Optimization of Set-Valued Variational In- equalities}

In this section we consider multiobjective optimization problems with equilibrium constraints described by set-valued variational inequalities of type (4); see Section 1 for more discussions. The main problem under consideration in this section is formulated as follows:

$$
\begin{array}{ll}
\text { minimize } & f(x, y) \\
\text { subject to } & \varphi_{i}(x, y) \leq 0, \quad i=1, \ldots, m, \\
& \varphi_{i}(x, y)=0, \quad i=m+1, \ldots, m+r, \\
& (x, y) \in \Omega, \\
& y \in \Xi \text { such that } \exists y^{*} \in G(x, y) \text { satisfying } \\
& \left\langle y^{*}, u-y\right\rangle \geq 0 \text { for all } u \in \Xi,
\end{array}
$$

where $G: X \times Y \rightrightarrows Y^{*}$ and $\Xi \subset Y$ while $f, \varphi_{i}$, and $\Omega$ are the same as in the general MOEC problem (8). Recall that we understand the multiobjective "minimization" in (38) in the sense of the generalized order $(f, \Theta)$-optimality from Definition 3.1.

It is easy to observe that problem (38) can be treated as a particular case of (8) with the set-valued base $G: X \times Y \rightrightarrows Y^{*}$ and the field $Q$ in the extended generalized equation (7) defined as the normal cone of convex analysis to the set $\Xi \subset Y$, i.e., as

$Q(y)=N(y ; \Xi)=\partial \delta(y ; \Xi):= \begin{cases}\left\{y^{*} \in Y^{*} \mid\left\langle y^{*}, u-y\right\rangle \leq 0 \text { for all } u \in \Xi\right\} & \text { if } y \in \Xi, \\ \emptyset & \text { otherwise, }\end{cases}$

where $\delta(\cdot ; \Xi)$ stands for the indicator function of $\Xi$ equal to 0 on this set and $\infty$ otherwise.

To establish necessary optimality conditions in the generalized variational problem (38), we recall the following construction of the second-order subdifferential of an extended-realvalued function $\varphi: X \rightarrow \overline{\mathbb{R}}$ at $\bar{x}$ relative to $\bar{y} \in \partial \varphi(\bar{x})$ defined by

$$
\partial^{2} \varphi(\bar{x}, \bar{y})(u):=\left(D^{*} \partial \varphi\right)(\bar{x}, \bar{y})(u), \quad u \in X^{* *} .
$$

In the books in Refs. 7, 16 and the references therein, the reader can find more details, various calculus rules, and applications of this construction, which reduces to the classical Hessian in the case of $C^{2}$ functions $\varphi$.

For brevity we formulate in what follows only a counterpart of Theorem 3.3 with the enhanced nontriviality condition for the case of problem (38).

Theorem 5.1 (necessary conditions for multiobjective optimization over setvalued variational inequalities). Let $(\bar{x}, \bar{y})$ be a local $(f, \Theta)$-optimal solution to problem (38), where the spaces $X, Z, W$, and both $Y$ and $Y^{*}$ are Asplund; the latter is automatic when $Y$ is reflexive. Pick $\bar{w} \in G(\bar{x}, \bar{y}) \cap(-N(\bar{y} ; \Xi))$ and assume that the initial data of (38) meet all the hypotheses of Theorem 3.3 with $Q(x, y)=N(y ; \Xi)$. Then there are

$$
\lambda_{1}, \ldots, \lambda_{m+r}, z^{*}, u, x^{*}, y^{*}, x_{1}^{*}, y_{1}^{*}, \ldots, x_{m+r}^{*}, y_{m+r}^{*}, x_{G}^{*}, y_{G}^{*}, y_{\Xi}^{*}, x_{\Omega}^{*}, y_{\Omega}^{*}
$$


satisfying the relationships

$$
\begin{aligned}
& \lambda_{i} \geq 0, \quad i=1, \ldots, m, \quad \lambda_{i} \in \mathbb{R}, i=m+1, \ldots, m+r, \\
& z^{*} \in N(0 ; \Theta), \quad u \in Y^{* *}, \\
& \left(x^{*}, y^{*}\right) \in D^{*} f(\bar{x}, \bar{y})\left(z^{*}\right), \\
& \left(x_{i}^{*}, y_{i}^{*}\right) \in D^{*} E_{\varphi_{i}}(\bar{x}, \bar{y}, 0)\left(\lambda_{i}\right), i=1, \ldots, m, \\
& \left(x_{i}^{*}, y_{i}^{*}\right) \in D^{*} \varphi_{i}(\bar{x}, \bar{y})\left(\lambda_{i}\right), i=m+1, \ldots, m+r, \\
& \left(x_{G}^{*}, y_{G}^{*}\right) \in D^{*} G(\bar{x}, \bar{y}, \bar{w})(u), \\
& y_{\Xi}^{*} \in \partial^{2} \delta((\bar{y}, \bar{w}) ; \Xi)(u), \\
& \left(x_{\Omega}^{*}, y_{\Omega}^{*}\right) \in N((\bar{x}, \bar{y}) ; \Omega), \\
& \left(x^{*}, y^{*}\right)+\sum_{i=1}^{m+r}\left(x_{i}^{*}, y_{i}^{*}\right)+\left(x_{G}^{*}, y_{G}^{*}\right)+\left(0, y_{\Xi}^{*}\right)+\left(x_{\Omega}^{*}, y_{\Omega}^{*}\right)=0
\end{aligned}
$$

together with the complementary slackness conditions (19) and the enhanced nontriviality

$$
\left(\lambda_{1}, \ldots, \lambda_{m+r}, z^{*}, u\right) \neq 0
$$

Proof. This easily follows from Theorem 3.3 due to the description of the equilibrium constraints in $(38)$ via $Q(y)=\partial \delta(y ; \Xi)$ and the construction of the second-order subdifferential for the case of the (extended-real-valued) indicator function.

It is worth emphasizing that there are exact calculations of the second-order subdifferential $\partial^{2} \delta(\cdot ; \Xi)$ of the indicator function for remarkable classes of sets frequently encountered in optimization-related problems, especially in finite dimensions. We particularly refer the reader to Ref. 31, where such calculations are performed for indicators of convex polyhedra, and to Ref. 32, where calculations are done for some other important classes of functions that may not have a polyhedral structure.

\section{Problems of Bilevel Programming}

In this section we study various problems related to bilevel programming and derive results providing necessary optimality conditions for them based on the above approach and development. Let us first consider the following problem of parametric optimization involving the decision variable $y$ (under minimization) with the parameter variable $x$ :

$$
\text { minimize } \varphi(x, y) \text { subject to } y \in \Xi(x),
$$

where $\varphi: X \times Y \rightarrow \overline{\mathbb{R}}$ is the cost function and $\Xi: X \Rightarrow Y$ is the moving (parameterdependent) feasible solution set that may be given in more specific forms using, e.g., equality and inequality constraints; see below for the further consideration.

Problem (39) can be viewed as the lower-level problem of hierarchical optimization, with the parameterized set $S(x)$ of optimal solutions to (39). Then considering problem (1) formulated in Section 1 in the case of $S(\cdot)$ given as the optimal solution map to (39) with a scalar cost function $f$, we get an upper-level problem of bilevel programming. The multiobjective version of (1) discussed in Section 1 from the viewpoint of vector optimization can be treated in this case as a multiobjective bilevel program. 
On the other hand, for any fixed $x$, a first-order necessary condition for optimal solutions to (39) is given via our basic normals and (partial) subgradients from Section 2 as

$$
0 \in \partial_{y} \varphi(x, y)+N(y ; \Xi(x))
$$

under natural assumptions on the initial data, e.g., when $\varphi$ is locally Lipschitzian in $y, \Xi(x)$ is closed, and $Y$ is Asplund; see Proposition 5.3 in Ref. 7. Condition (40) is known to be also sufficient for optimality under certain convexity-type and qualification requirements. In general, it is conventional (see. e.g., Ref. 4) to replace the lower-level problem (39) by the generalized equation (40) written in the extended form (7), with both base and field mappings being set-valued whenever the cost function $\varphi$ is nonsmooth in $y$. Thus upper level problems of (multiobjective) bilevel programming reduce to the basic MOEC problem (8) studied in this paper with

$$
G(x, y):=\partial_{y} \varphi(x, y): X \times Y \rightrightarrows Y^{*} \text { and } Q(x, y):=N(y ; \Xi(x)) .
$$

Applying now the results for general MOECs from Section 3 to the case of (41), we arrive at necessary optimality conditions for multiobjective bilevel programs with the corresponding inclusions for $\left(x_{G}^{*}, y_{G}^{*}\right)$ and $\left(x_{Q}^{*}, y_{Q}^{*}\right)$ in $(18)$ written as

$$
\left(x_{G}^{*}, y_{G}^{*}\right) \in\left(D^{*} \partial_{y} \varphi\right)(\bar{x}, \bar{y}, \bar{w})\left(w^{*}\right), \quad\left(x_{Q}^{*}, y_{Q}^{*}\right) \in\left(D^{*} N(\cdot ; \Xi(\cdot))\right)(\bar{x}, \bar{y},-\bar{w})\left(w^{*}\right)
$$

where $\bar{w} \in \partial_{y} \varphi(\bar{x}, \bar{y}) \cap\left(-N(\bar{y} ; \Xi(\bar{x}))\right.$ and $w^{*} \in Y^{* *}$. Note that the constructions $D^{*} \partial_{y} \varphi$ and $D^{*} N(\cdot ; \Xi(x))$ in (42) reduce to the partial second-order subdifferentials of $\varphi$ and of the indicator function to $\Xi(x)$, respectively, for which some calculus rules and applications to parametric optimization were developed in Ref. 33.

Let the feasible constraint mapping $\Xi(\cdot)$ in (39) be given in the more specific form

$$
\begin{aligned}
& \Xi(x):=\left\{y \in Y \mid \psi_{j}(x, y) \leq 0 \text { for } j=1, \ldots, l,\right. \\
& \left.\psi_{j}(x, y)=0 \text { for } j=l+1, \ldots, l+p, \quad y \in \Lambda\right\},
\end{aligned}
$$

where we consider for simplicity the case of smooth functions $\psi_{j}$ with respect to the decision variable $y$. Furthermore, assume that the cost function $\varphi$ in (39) is smooth with respect to $y$ as well around the reference points. Then the corresponding lower-level system (40) in bilevel programming, unified with the above description of the constraints in (39), reduces to the (generalized) Karush-Kuhn-Tucker (KKT) system

$$
\begin{aligned}
& 0 \in \nabla_{y} \varphi(x, y)+\sum_{j=1}^{l+p} \mu_{j} \nabla_{y} \psi_{j}(x, y)+N(y ; \Lambda), \\
& \psi_{j}(x, y) \leq 0 \text { for } j=1, \ldots, l, \\
& \psi_{j}(x, y)=0 \text { for } j=l+1, \ldots, l+p, \\
& \mu_{j} \geq 0 \text { and } \mu_{j} \psi_{j}(x, y)=0 \text { for } j=1, \ldots, l
\end{aligned}
$$

with some multipliers $\mu=\left(\mu_{1}, \ldots, \mu_{l+p}\right) \in \mathbb{R}^{l+p}$. Denote

$$
\psi(x, y):=\left(\psi_{1}(x, y), \ldots, \psi_{l+p}(x, y)\right), \quad\left\langle\mu, \nabla_{y} \psi(x, y)\right\rangle:=\sum_{j=1}^{l+p} \mu_{j} \nabla_{y} \psi_{j}(x, y)
$$


(in what follows we will use this convenient inner product notation for the multiplication of vectors and operators) and consider the single-valued mapping $g: X \times Y \times \mathbb{R}^{l+p} \rightarrow Y^{*} \times \mathbb{R}^{l+p}$ and the set-valued mapping $Q: Y \times \mathbb{R}^{l+p} \rightrightarrows Y^{*} \times \mathbb{R}^{l+p}$ defined by

$g(x, y, \mu):=\left(\nabla_{y} \varphi(x, y)+\left\langle\mu, \nabla_{y} \psi(x, y)\right\rangle,-\psi(x, y)\right), \quad Q(y, \mu):=N\left((y, \mu) ; \Lambda \times \mathbb{R}_{\geq}^{l} \times \mathbb{R}^{p}\right)$.

Then the KKT system (43) can be written as the generalized equation

$$
0 \in g(x, y, \mu)+Q(y, \mu) .
$$

Now we consider the multiobjective optimization problem in (8) with the equilibrium constraints given by (44) assuming for simplicity that the cost mapping $f$ and the constraint functions $\varphi_{i}$ in (8) are strictly differentiable at the reference optimal solution together with the base mapping $g$ in (44).

Theorem 6.1 (necessary optimality conditions for multiobjective bilevel programs). Let $(\bar{x}, \bar{y})$ be a local $(f, \Theta)$-optimal solution to $M O E C(8)$ with the equilibrium constraints (44), let $\mu=\left(\mu_{1}, \ldots, \mu_{l+p}\right)$ be the corresponding KKT vector from (43), and let

$$
\bar{w}:=\nabla_{y} \varphi(\ddot{x}, \bar{y})+\left\langle\mu \nabla_{y} \psi(\bar{x}, \bar{y})\right\rangle .
$$

Assume that $X$ and $Z$ are Asplund while $\operatorname{dim} Y<\infty$, that the sets $\Theta$ and $\Omega$ are locally closed around 0 and $(\bar{x}, \bar{y})$, respectively, and that the mappings $f, \varphi_{i}, \psi_{j}, \nabla_{y} \varphi_{i}$, and $\nabla_{y} \psi_{j}$ are strictly differentiable at $(\bar{x}, \bar{y})$ for all $i=1, \ldots, m+r$ and $j=1, \ldots, l+p$. Then there are nontrivial dual elements

$$
\left(\lambda_{1}, \ldots, \lambda_{m+r}, z^{*}, u, \nu\right) \neq 0 \text { with } \nu:=\left(\nu_{1}, \ldots, \nu_{l+p}\right)
$$

such that the following optimality conditions are satisfied:

$$
\begin{aligned}
& \lambda_{i} \geq 0, i=1, \ldots, m, \quad \lambda_{i} \in \mathbb{R}, i=m+1, \ldots, m+r \\
& z^{*} \in N(0 ; \Theta), \quad u \in Y, \\
& \lambda_{i} \varphi_{i}(\bar{x}, \bar{y})=0, \quad i=1, \ldots, m+r \\
& \nu_{j} \geq 0, j=1, \ldots, l, \quad \nu_{j} \in \mathbb{R}, j=l+1, \ldots, l+p \\
& \left(-\nabla_{y} \psi(\vec{x}, \bar{y}), \nu\right) \in \partial^{2} \delta\left(\cdot ; \Lambda \times \mathbb{R}_{\geq}^{l} \times \mathbb{R}^{p}\right)(\bar{y}, \mu,-\bar{w}, \psi(\bar{x}, \bar{y})), \quad \text { and } \\
& 0 \in \nabla f(\ddot{x}, \bar{y}) z^{*}+\sum_{i=1}^{m+r} \lambda_{i} \nabla \varphi_{i}(\bar{x}, \bar{y})+\left[\nabla\left(\nabla_{y} \varphi+\left\langle\mu, \nabla_{y} \psi\right\rangle(\bar{x}, \bar{y})\right)\right]^{*} u \\
& -\langle\nu, \nabla \psi\rangle(\bar{x}, \bar{y})+\left(0,-\nabla_{y} \psi(\bar{x}, \bar{y})^{*} u\right)+N((\bar{x}, \bar{y}) ; \Omega) .
\end{aligned}
$$

Proof. This follows directly from the necessary conditions of Theorem 3.2 applied to the MOEC problem (8), (44) under consideration due to the equilibrium constraint structure in (8) and by the assumptions imposed in the theorem, which ensure the fulfillment of those in Theorem 3.2. The reader can check all the details based on the corresponding results reviewed in Section 2; see also Ref. 16.

Observe that our approach and results allow us to avoid many troubles in deriving necessary optimality conditions in problems of bilevel programming; in particular, those related to the violation of conventional qualification conditions at the upper level like MangasarianFromovitz constraint qualifications, etc.; compare, e.g., Refs. 1, 2, 4, 11, 14 among other publications. Note also that the second-order subgradients of the indicator function in Theorem 6.1 can be efficiently calculated for favorable settings overwhelmingly involved into finite-dimensional optimization; see the discussion and references after Theorem 5.1. 


\section{Illustrative Example}

In the final section of the paper, we illustrate the scheme of applications of the results obtained by the example from nondifferentiable bilevel programming, which reduces to the MOEC problem (8) governed by the extended generalized equations (7).

Example 7.1 (computing optimal solutions to a nondifferentiable bilevel program). Consider the one-dimensional bilevel program:

$$
\begin{aligned}
& \operatorname{minimize}|x|+|y|, \quad x, y \in \mathbb{R} \\
& \text { subject to } y \in \operatorname{argmin}\left\{\frac{1}{2}|x| y^{2}+|y| \mid y \in[-1,1]\right\}
\end{aligned}
$$

Note that the lower-level minimization problem is a convex one, so it is equivalent to the extended generalized equation

$$
0 \in \partial_{y}\left(\frac{1}{2}|x| y^{2}+|y|\right)+N(y ;[-1,1]) \text {. }
$$

Define the set-valued mappings $G: \mathbb{R}^{2} \Rightarrow \mathbb{R}$ and $Q: \mathbb{R} \Rightarrow \mathbb{R}$ by

$$
\begin{gathered}
G(x, y):=\partial_{y}\left(\frac{1}{2}|x| y^{2}+|y|\right)=\left\{\begin{array}{l}
|x| y+1, y>0, \\
|x| y-1 \quad y<0, \\
{[-1,1] y=0}
\end{array}\right. \\
Q(y):=N(y ;[-1,1])=\left\{\begin{array}{l}
(\infty, 0], y=-1, \\
0,-1<y<1, \\
{[0, \infty), y=1}
\end{array}\right.
\end{gathered}
$$

The bilevel programming problem under consideration is equivalent to MOEC (8) with the cost function $f(x, y):=|x|+|y|$, the sets $\Theta=\mathbb{R}_{-}, \Omega=\mathbb{R}^{2}$, and the other data defined above. Observe that the point $(0,0)$ satisfies the necessary optimality condition of the main Theorem 3.2, since we have $\partial f(0,0)=[-1,1] \times[-1,1]$ and thus

$$
(0,0) \in \partial f(0,0)+D^{*} G(0,0,0)(0)+\{0\} \times D^{*} Q(0,0)(0) .
$$

Further, take any $x \neq 0$ and $y \neq 0$ and observe that there is no $w \in G(x, y) \cap(-Q(y))$. Hence the point $(x, y)$ with $x \neq 0$ is not a feasible solution.

If $x=0$, then we have

$$
\begin{gathered}
G(0, y)=\left\{\begin{array}{l}
1, \quad y>0 \\
-1, \quad y<0 \\
{[-1,1], \quad y=0}
\end{array}\right. \\
Q(y)=\left\{\begin{array}{l}
(\infty, 0], \quad y=-1, \\
0,-1<y<1 \\
{[0, \infty), \quad y=1}
\end{array}\right.
\end{gathered}
$$

This shows that there is no $y \neq 0$ such that $G(0, y) \cap(-Q(y)) \neq \emptyset$, which rules out such points from those suspicious for optimality. 
It remains to consider the case of $x \neq 0$ and $y=0$. Then we have $G(x, 0)=[-1,1]$ and $Q(0)=\{0\}$, and thus $0 \in G(x, 0) \cap(-Q(0))$. It is easy to compute that

$$
\begin{gathered}
\partial(|x|+|y|)(x, 0)=\left\{\begin{array}{l}
1 \times[-1,1], \quad x>0, \\
-1 \times[-1,1], \quad x<0,
\end{array}\right. \\
D^{*} G(x, 0,0)(u)=\{0\} \times \mathbb{R}, \text { and } D^{*} Q(x, 0)(u)=\{0\} \text { for all } u \in \mathbb{R} .
\end{gathered}
$$

Thus such points $(x, 0)$ do not satisfy the conclusions of Theorem 3.2 and hence cannot be optimal to the problem under consideration. Summarizing, we conclude that the pair $(0,0)$ is the only point satisfying the necessary optimality conditions of the theorem. In fact, this point is the unique optimal solution to the above bilevel program.

\section{References}

1. Luo, Z.-Q., PANG, J.-S., and RALPH, D., Mathematical Programs with Equilibrium Constraints, Cambridge University Press, Cambridge, UK, 1996.

2. Outrata, J. V., Kočvara, M., and Zowe, J., Nonsmooth Approach to Optimization Problems with Equilibrium Constraints, Kluwer Academic Publishers, Dordrecht, The Netherlands, 1998.

3. Giannessi, F., Editor, Vector Variational Inequalities and Vector Equilibria, Kluwer Academic Publishers, Dordrecht, The Netherlands, 2000.

4. DemPe, S., Foundations of Bilevel Programming, Kluwer Academic Publishers, Dordrecht, The Netherlands, 2002.

5. FACChINeI, F., and PANG, J.-S., Finite-Dimensional Variational Inequalities and Complementarity Problems, Springer, New York, 2003.

6. Giannessi, F., Constrained Optimization and Image Space Analysis, I: Separation of Sets and Optimality Conditions, Springer, New York, 2005.

7. MordukHovich, B. S., Variational Analysis and Generalized Differentiation, II: Applications, Springer, Berlin, 2006.

8. RoBinson, S. M., Generalized Equations and Their Solutions, I: Basic Theory, Mathematical Programming Study, Vol. 10, pp. 128-141, 1979.

9. Zhang, R., Problems of Hierarchical Optimization in Finite Dimensions, SIAM Journal on Optimization, Vol. 4, pp. 521-536, 1994.

10. YE, J. J., and Ye, X. Y., Necessary Optimality Conditions for Optimization Problems with Variational Inequality Constraints, Mathematics of Operations Research, Vol. 22, pp. 977-997, 1997.

11. Outrata, J. V., Optimality Conditions for a Class of Mathematical Programs with Equilibrium Constraints, Mathematics of Operations Research, Vol. 24, pp. 627-644, 1999.

12. Outrata, J. V., A Generalized Mathematical Program with Equilibrium Constraints, SIAM Journal on Control and Optimization, Vol. 38, pp. 1623-1638, 2000.

13. Mondukhovich, B. S., Necessary Conditions in Nonsmooth Minimization via Lower and Upper Subgradients, Set-Valued Analysis, Vol. 12, pp. 163-193, 2004.

14. YE, J. J., Necessary and Sufficient Optimality Conditions for Mathematical Programs with Equilibrium Constraints, Journal of Mathematical Analysis and Applications, Vol. 307, pp. 350-369, 2005. 
15. Flegel, M. L., and Kanzow, C., On M-Stationarity Points for Mathematical Programs with Equilibrium Constraints, Journal of Mathematical Analysis and Applications, Vol. 310, pp. 286-302, 2005.

16. MordukHovich, B. S., Variational Analysis and Generalized Differentiation, I: Basic Theory, Springer, Berlin, 2006.

17. YE, J. J., and ZHU, Q. J., Multiobjective Optimization Problem with Variational Inequality Constraints, Mathematical Programming, Vol. 96, pp. 139-160, 2003.

18. MoRduKhovich, B. S., Equilibrium Problems with Equilibrium Constraints via Multiobjective Optimization, Optimization Methods and Software, Vol. 19, pp. 479492, 2004.

19. Mordukhovich, B. S., Outrata, J. V., and Červinka, M., Equilibrium Problems with Complementarity Constraints: Case Study with Applications to Oligopolistic Markets, to appear in Optimization.

20. Outrata, J. V., A Note on a Class of Equilibrium Problems with Equilibrium Constraints, Kybernetika, Vol. 40, pp. 585-594, 2004.

21. Aussel, D., and HadjISAVVAs, N., On Quasimonotone Variational Inequalities, Journal of Optimization Theory and Applications, Vol. 121, pp. 445-450, 2004.

22. Robinson, S. M., Variational Conditions with Smooth Constraints: Structure and Analysis, Mathematical Programming, Vol. 97, pp. 245-265, 2003.

23. Rockafellar, R. T., and Wets, R. J.-B., Variational Analysis, Springer, Berlin, 1998.

24. Borwern, J. M., and ZHU, Q. J., Techniques of Variational Analysis, Springer, New York, 2005.

25. Phelps, R. R., Convex Functions, Monotone Operators and Differentiability, Springer, Berlin, 1993.

26. Ioffe, A. D., Optimality Alternative: A Non-Variational Approach to Necessary Conditions, in Variational Analysis and Applications (Giannessi, F., and Maugeri, A., Editors), pp. 531-552, Springer, Berlin, 2005.

27. Borwein, J. M., and Strójwas, H. M., Tangential Approximations, Nonlinear Analysis, Vol. 9, pp. 1347-1366, 1985.

28. Fabian, M., and Mordukhovich, B. S., Sequential Normal Compactness Versus Topological Normal Compactness in Variational Analysis, Nonlinear Analysis, Vol. 54, pp. 1057-1067, 2003.

29. Mordukhovich, B. S., Treiman, J. S., and ZhU, Q. J., An Extended Extremal Principle with Applications to Multiobjective Optimization, SIAM Journal on Optimization, Vol. 14, pp. 359-379, 2003.

30. Gupta, P., ShIraIshi, S., and YokoYama, K., E-Optimality without Constraint Qualifications for Multiobjective Fractional Programs, Journal of Nonlinear and Convex Analysis, Vol. 6, pp. 347-557, 2005.

31. Dontchev, A. L., and Rockafellar, R. T., Characterizations of Strong Regularity for Variational Inequalities over Polyhedral Convex Sets, SIAM Journal on Optimization, Vol. 7, pp. 1087-1105, 1996.

32. Mordukhovich, B. S., and Outrata, J. V., Second-Order Subdifferentials and Their Applications, SIAM Journal on Optimization, Vol. 12, pp. 139-169, 2001.

33. Levy, A. B., and Mordukhovich, B. S., Coderivatives in Parametric Optimization, Mathematical Programming, Vol 99, pp. 311-327, 2004. 